\title{
Equilibria of homogeneous functionals in the fair-competition regime
}

\author{
V. Calvez ${ }^{\text {a }}$, J.A. Carrillo ${ }^{\text {b,* }}$, F. Hoffmann ${ }^{c, b}$ \\ ${ }^{a}$ Unité de Mathématiques Pures et Appliquées, CNRS UMR 5669 and équipe-projet INRIA NUMED, École \\ Normale Supérieure de Lyon, Lyon, France \\ ${ }^{\mathrm{b}}$ Department of Mathematics, Imperial College London, South Kensington Campus, London SW7 2AZ, UK \\ ${ }^{\mathrm{c}}$ DPMMS, Centre for Mathematical Sciences, University of Cambridge, Wilberforce Road, Cambridge CB3 \\ $O W A, U K$
}

\section{A R T I C L E I N F O}

\section{Article history:}

Received 4 October 2016

Accepted 16 March 2017

Communicated by S. Carl

\section{Keywords:}

Aggregation-diffusion equations

Gradient flows

Minimisation

\begin{abstract}
A B S T R A C T
We consider macroscopic descriptions of particles where repulsion is modelled by non-linear power-law diffusion and attraction by a homogeneous singular/ non-singular kernel leading to variants of the Keller-Segel model of chemotaxis. We analyse the regime in which both homogeneities scale the same with respect to dilations, that we coin as fair-competition. In the singular kernel case, we show that existence of global equilibria can only happen at a certain critical value and they are characterised as optimisers of a variant of HLS inequalities. We also study the existence of self-similar solutions for the sub-critical case, or equivalently of optimisers of rescaled free energies. These optimisers are shown to be compactly supported radially symmetric and non-increasing stationary solutions of the nonlinear Keller-Segel equation. On the other hand, we show that no radially symmetric non-increasing stationary solutions exist in the non-singular kernel case, implying that there is no criticality. However, we show the existence of positive self-similar solutions for all values of the parameter under the condition that diffusion is not too fast. We finally illustrate some of the open problems in the non-singular kernel case by numerical experiments.
\end{abstract}

๑) 2017 The Author(s). Published by Elsevier Ltd. This is an open access article under the CC BY license (http://creativecommons.org/licenses/by/4.0/).

\section{Introduction}

The goal of this work is to investigate properties of the following class of homogeneous functionals, defined for centred probability densities $\rho(x)$, belonging to suitable $L^{p}$-spaces, and some interaction strength

\footnotetext{
* Corresponding author.

E-mail addresses: vincent.calvez@ens-lyon.fr (V. Calvez), carrillo@imperial.ac.uk (J.A. Carrillo), fkoh2@cam.ac.uk (F. Hoffmann).
} 
coefficient $\chi>0$ and diffusion power $m>0$ :

$$
\begin{aligned}
\mathcal{F}_{m, k}[\rho] & =\int_{\mathbb{R}^{N}} U_{m}(\rho(x)) d x+\chi \iint_{\mathbb{R}^{N} \times \mathbb{R}^{N}} \rho(x) W_{k}(x-y) \rho(y) d x d y \\
& :=\mathcal{U}_{m}[\rho]+\chi \mathcal{W}_{k}[\rho], \\
& \rho(x) \geq 0, \quad \int_{\mathbb{R}^{N}} \rho(x) d x=1, \quad \int_{\mathbb{R}^{N}} x \rho(x) d x=0,
\end{aligned}
$$

with

$$
U_{m}(\rho)= \begin{cases}\frac{1}{N(m-1)} \rho^{m}, & \text { if } m \neq 1 \\ \frac{1}{N} \rho \log \rho, & \text { if } m=1\end{cases}
$$

and

$$
W_{k}(x)= \begin{cases}\frac{|x|^{k}}{k}, & \text { if } k \in(-N, N) \backslash\{0\} \\ \log |x|, & \text { if } k=0 .\end{cases}
$$

The conditions on $k$ imply that the kernel $W_{k}(x)$ is locally integrable in $\mathbb{R}^{N}$. The centre of mass is assumed to be zero since the free energy functional is invariant by translation.

There exists a strong link between the aforementioned functional (1.1) and the following family of partial differential equations modelling self-attracting diffusive particles at the macroscopic scale,

$$
\left\{\begin{array}{l}
\partial_{t} \rho=\frac{1}{N} \Delta \rho^{m}+2 \chi \nabla \cdot\left(\rho \nabla S_{k}\right), \quad t>0, x \in \mathbb{R}^{N}, \\
\rho(t=0, x)=\rho_{0}(x) \geq 0, \quad \int_{\mathbb{R}^{N}} \rho_{0}(x) d x=1, \quad \int_{\mathbb{R}^{N}} x \rho_{0}(x) d x=0,
\end{array}\right.
$$

where we define the mean-field potential $S_{k}(x):=W_{k}(x) * \rho(x)$. For $k>1-N$, the gradient $\nabla S_{k}:=$ $\nabla\left(W_{k} * \rho\right)$ is well defined. For $-N<k \leq 1-N$ however, it becomes a singular integral, and we thus define it via a Cauchy principal value. Hence, the mean-field potential gradient in Eq. (1.2) is given by

$$
\nabla S_{k}(x):= \begin{cases}\nabla W_{k} * \rho, & \text { if } k>1-N, \\ \int_{\mathbb{R}^{N}} \nabla W_{k}(x-y)(\rho(y)-\rho(x)) d y, & \text { if }-N<k \leq 1-N .\end{cases}
$$

The noticeable characteristic of the class of PDEs (1.2) and the functional $\mathcal{F}_{m, k}$ consists in the competition between the diffusion (possibly non-linear), and the non-local, quadratic non-linearity which is due to the self-attraction of the particles through the mean-field potential $S_{k}$. The parameter $\chi>0$ measures the strength of the interaction and scales with the mass of solution densities.

The strong connection between the functional $\mathcal{F}_{m, k}$ and the PDE (1.2) is due to the fact that the functional $\mathcal{F}_{m, k}$ is non-increasing along the trajectories of the system. Namely $\mathcal{F}_{m, k}$ is the free energy of the system and it satisfies at least formally

$$
\frac{d}{d t} \mathcal{F}_{m, k}[\rho(t)]=-\int_{\mathbb{R}^{N}} \rho(t, x)\left|\nabla\left(\frac{m}{N(m-1)} \rho(t, x)^{m-1}+2 \chi W_{k}(x) * \rho(t, x)\right)\right|^{2} d x .
$$

Furthermore, the system (1.2) is the formal gradient flow of the free energy functional (1.1) when the space of probability measures is endowed with the Euclidean Wasserstein metric W. This means that the family of PDEs (1.2) can be written as

$$
\partial_{t} \rho(t)=\nabla \cdot\left(\rho(t) \nabla \mathcal{T}_{m, k}[\rho(t)]\right)=-\nabla_{\mathbf{W}} \mathcal{F}_{m, k}[\rho(t)],
$$

where $\mathcal{T}_{m, k}[\rho]$ denotes the first variation of the energy functional in the set of probability densities:

$$
\mathcal{T}_{m, k}[\rho](x):=\frac{\delta \mathcal{F}_{m, k}}{\delta \rho}[\rho](x)=\frac{m}{N(m-1)} \rho^{m-1}(x)+2 \chi W_{k}(x) * \rho(x) .
$$


This illuminating statement has been clarified in the seminal paper by Otto [42] for the porous medium equation, and generalised to a large family of equations subsequently in [21,1,22], we refer to [55,1] for a comprehensive presentation of this theory of gradient flows in Wasserstein metric spaces, particularly in the convex case. Let us mention that such a gradient flow can be constructed as the limit of discrete in time steepest descent schemes. Performing gradient flows of a convex functional is a natural task, and suitable estimates from below on the right notion of Hessian of $\mathcal{F}_{m, k}$ translate into a rate of convergence towards equilibrium for the PDE [55,21,1]. However, performing gradient flows of non-convex functionals is much more delicate, and one has to seek compensations. Such compensations do exist in our case, and we will observe them at the level of existence of minimisers for the free energy functional $\mathcal{F}_{m, k}$ and stationary states of the family of PDEs (1.2) in particular regimes.

The family of non-local problems (1.2) has been intensively studied in various contexts arising in physics and biology. The two-dimensional logarithmic case $(m=1, k=0)$ is the so-called Keller-Segel system in its simplest formulation $[35,36,41,33,31,9,43]$. It has been proposed as a model for chemotaxis in cell populations. Cells may interact with each other by secreting a chemical substance to attract cells around them. This occurs for instance during the starvation stage of the slime mould Dictyostelium discoideum. More generally, chemotaxis is widely observed in various biological fields (morphogenesis, bacterial self-organisation, inflammatory processes among others). The two- and three-dimensional configurations with Newtonian interaction $(m=1, k=2-N)$ are the so-called Smoluchowski-Poisson system arising in gravitational physics. It describes macroscopically a density of particles subject to a self-sustained gravitational field [24,25].

Let us describe in more detail the two-dimensional Keller-Segel system as the analysis of its peculiar structure will serve as a guideline to understand the other cases. In fact, the functional $(1.1)(m=1, k=0)$ is bounded from below if and only if $\chi=1$. The gradient flow is also subject to a remarkable dichotomy, well described mathematically. The density exists globally in time if $\chi<1$ (diffusion overcomes self-attraction), whereas blow-up occurs in finite time when $\chi>1$ (self-attraction overwhelms diffusion). This transition has been first formulated in [28]. Mathematical contributions are [33] for the existence part, [40] for the radial case, and [31,9] in the full space. The critical case $\chi=1$ was analysed further in $[8,6,16]$ in terms of stability of stationary states.

The effect of substituting linear diffusion by non-linear diffusion with $m>1$ in two dimensions and higher was described in $[11,51]$ where it is shown that solutions exist globally in time for all values of the parameter $\chi>0$. The role of both non-linear diffusion and non-local aggregation terms was clarified in [7], see also [50], where the authors find that there is a similar dichotomy to the two-dimensional classical Keller-Segel case $(N=2, m=1, k=0)$, for a whole range of parameters, choosing the non-local term as the Newtonian potential, $(N \geq 3, m=2-2 / N, k=2-N)$. The main difference is that the stationary states found for the critical case are compactly supported. Choosing the non-local term as the Newtonian potential, this range of parameters can be understood as fixing the non-linear diffusion such that both terms in the functional $\mathcal{F}_{m, k}$ scale equally for mass-preserving dilations. This mass-preserving dilation homogeneity of the functional $\mathcal{F}_{m, k}$ is shared by the range of parameters $(m, k)$ with $N(m-1)+k=0$ for all dimensions, $m>0$ and $k \in(-N, N)$. We call this range of parameters the fair-competition regime, since both terms are competing each other at equal foot.

We will analyse the properties of the functional $\mathcal{F}_{m . k}$ in relation to global minimisers and its relation to stationary states of (1.2) in this work. We will first define properly the notion of stationary states to (1.2) and analyse their basic properties in Section 2. We will also state and explain the main results of this work once the different regimes have been introduced. We postpone further discussion of the related literature to Section 2. Section 3 is devoted to the fair-competition regime with $k<0$ for which we show a similar dichotomy to [7] in the whole range $k \in(-N, 0)$ including the most singular cases. We show that stationary states exist only for a critical value of $\chi$ and that they are compactly supported, bounded, radially symmetric decreasing and continuous functions. Moreover, we show that they are global minimisers of $\mathcal{F}_{m . k}$. The 
sub-critical case is also analysed in scaled variables and we show the existence of global minimisers with the properties above leading to the existence of self-similar solutions in original variables. The critical parameter is characterised by a variant of HLS inequalities as in [7]. Let us mention that the regularity results need a careful treatment of the problem in radial coordinates involving non-trivial properties of hypergeometric functions. The properties of the kernel in radial coordinates are postponed to the Appendix.

In Section 4, we analyse the case $k>0$. Let us mention that there are no results in the literature to our knowledge concerning the case $k \in(0, N)$ in which $0<m=1-k / N<1$. There is one related result in [29] for the limiting case in one dimension taking $m=0$, corresponding to logarithmic diffusion, and $k=1$. They showed that no criticality is present in that case as solutions to $(1.2)$ with $(m=0, k=1)$ are globally defined in time for all values of the parameter $\chi>0$. We show that no radially symmetric non-increasing stationary states and no radially symmetric non-increasing global minimisers exist in original variables for all values of the critical parameter $\chi$ and for $k \in(0, N)$ while we show the existence of stationary states for all values of the critical parameter $\chi$ in scaled variables for $k \in(0,1]$. In this sense, we show that there is no criticality for $k>0$. A full proof of non-criticality involves the analysis of the minimisation problem in scaled variables as for $k<0$ showing that global minimisers exist in the right functional spaces for all values of the critical parameter and that they are indeed stationary states. This is proven in one dimension in [13] by optimal transport techniques and postponed for further future investigations in general dimension. We finally illustrate these results by numerical experiments in one dimension corroborating the absence of critical behaviour for $k>0$.

\section{Stationary states \& main results}

\subsection{Stationary states: Definition $\&$ basic properties}

Let us define precisely the notion of stationary states to the aggregation equation (1.2).

Definition 2.1. Given $\bar{\rho} \in L_{+}^{1}\left(\mathbb{R}^{N}\right) \cap L^{\infty}\left(\mathbb{R}^{N}\right)$ with $\|\bar{\rho}\|_{1}=1$, it is a stationary state for the evolution equation (1.2) if $\bar{\rho}^{m} \in \mathcal{W}_{\text {loc }}^{1,2}\left(\mathbb{R}^{N}\right), \nabla \bar{S}_{k} \in L_{l o c}^{1}\left(\mathbb{R}^{N}\right)$, and it satisfies

$$
\frac{1}{N} \nabla \bar{\rho}^{m}=-2 \chi \bar{\rho} \nabla \bar{S}_{k}
$$

in the sense of distributions in $\mathbb{R}^{N}$. If $-N<k \leq 1-N$, we further require $\bar{\rho} \in C^{0, \alpha}\left(\mathbb{R}^{N}\right)$ with $\alpha \in(1-k-N, 1)$.

We start by showing that the function $S_{k}$ and its gradient defined in (1.3) satisfy even more than the regularity $\nabla S_{k} \in L_{l o c}^{1}\left(\mathbb{R}^{N}\right)$ required in Definition 2.1 .

Lemma 2.2. Let $\rho \in L_{+}^{1}\left(\mathbb{R}^{N}\right) \cap L^{\infty}\left(\mathbb{R}^{N}\right)$ with $\|\rho\|_{1}=1$. If $0<k<N$, we additionally assume $|x|^{k} \rho \in L^{1}\left(\mathbb{R}^{N}\right)$. Then the following regularity properties hold:

(i) $S_{k} \in L_{\text {loc }}^{\infty}\left(\mathbb{R}^{N}\right)$ for $0<k<N$ and $S_{k} \in L^{\infty}\left(\mathbb{R}^{N}\right)$ for $-N<k<0$.

(ii) $\nabla S_{k} \in L_{\text {loc }}^{\infty}\left(\mathbb{R}^{N}\right)$ for $1<k<N$ and $\nabla S_{k} \in L^{\infty}\left(\mathbb{R}^{N}\right)$ for $-N<k<0$ and $0<k \leq 1$, assuming additionally $\rho \in C^{0, \alpha}\left(\mathbb{R}^{N}\right)$ with $\alpha \in(1-k-N, 1)$ in the range $-N<k \leq 1-N$.

Proof. A direct decomposition in near- and far-field sets $\mathcal{A}:=\{y:|x-y| \leq 1\}$ and $\mathcal{B}:=\mathbb{R}^{N}-\mathcal{A}$ yields for $-N<k<0$ and $x \in \mathbb{R}^{N}$, 


$$
\begin{aligned}
\left|S_{k}(x)\right| & \leq \int_{\mathbb{R}^{N}}\left|W_{k}(x-y)\right| \rho(y) d y \leq \frac{1}{|k|} \int_{\mathcal{A}}|x-y|^{k} \rho(y) d y+\frac{1}{|k|} \int_{\mathcal{B}} \rho(y) d y \\
& \leq \frac{1}{|k|}\left(\frac{\sigma_{N}}{k+N}\|\rho\|_{\infty}+1\right)<\infty,
\end{aligned}
$$

where $\sigma_{N}=2 \pi^{(N / 2)} / \Gamma(N / 2)$ denotes the surface area of the $N$-dimensional unit ball. Locally, boundedness extends to the fast diffusion regime $0<k<N$ by using the inequality

$$
|x-y|^{k} \leq \eta\left(|x|^{k}+|y|^{k}\right), \quad \eta=\max \left\{1,2^{k-1}\right\} .
$$

This inequality follows directly from splitting into cases $k<1$ and $k>1$. The inequality $|x-y|^{k} \leq|x|^{k}+|y|^{k}$ is true for any $k \in(0,1]$ with $x, y \in \mathbb{R}^{N}$ by direct inspection. For $N>1$ and $k \in(1, N)$, we have by convexity $|x-y|^{k} \leq 2^{k-1}\left(|x|^{k}+|y|^{k}\right)$, for any $x, y \in \mathbb{R}^{N}$, and so (2.2) holds true.

Similarly, in order to prove (ii) for $1-N<k \leq 1$ and $x \in \mathbb{R}^{N}$, we estimate $\nabla S_{k}$ as

$$
\begin{aligned}
\left|\nabla S_{k}(x)\right| & \leq \int_{\mathbb{R}^{N}}\left|\nabla W_{k}(x-y)\right| \rho(y) d y \leq \int_{\mathcal{A}}|x-y|^{k-1} \rho(y) d y+\int_{\mathcal{B}} \rho(y) d y \\
& \leq\left(\frac{\sigma_{N}}{k+N-1}\|\rho\|_{\infty}+1\right)<\infty .
\end{aligned}
$$

In the Cauchy integral range $-N<k \leq 1-N$, we additionally require a certain Hölder regularity, yielding

$$
\begin{aligned}
\left|\nabla S_{k}(x)\right| & =\left|\int_{\mathcal{A}} \nabla W_{k}(x-y)(\rho(y)-\rho(x)) d y+\int_{\mathcal{B}} \nabla W_{k}(x-y)(\rho(y)-\rho(x)) d y\right| \\
& \leq \int_{\mathcal{A}}\left|\nabla W_{k}(x-y)\right||\rho(y)-\rho(x)| d y+\int_{\mathcal{B}}\left|\nabla W_{k}(x-y)\right| \rho(y) d y \\
& \leq[\rho]_{\alpha} \int_{\mathcal{A}}|x-y|^{k-1}|x-y|^{\alpha} d y+\int_{\mathcal{B}} \rho(y) d y<\infty,
\end{aligned}
$$

where $[\rho]_{\alpha}$ denotes the $\alpha$-Hölder semi-norm of $\rho$, and where the term $\int_{\mathcal{B}} \nabla W_{k}(x-y) d y$ vanishes by antisymmetry. For $1<k<N$ and $x$ in some compact set, we have

$$
\begin{aligned}
\left|\nabla S_{k}(x)\right| & \leq \int_{\mathcal{A}}|x-y|^{k-1} \rho(y) d y+\int_{\mathcal{B}}|x-y|^{k-1} \rho(y) d y \\
& \leq \frac{\sigma_{N}}{k+N-1}\|\rho\|_{\infty}+\int_{\mathcal{B}}|x-y|^{k} \rho(y) d y
\end{aligned}
$$

which concludes $\nabla S_{k} \in L_{l o c}^{\infty}\left(\mathbb{R}^{N}\right)$ using (2.2) and the fact that the $k$ th moment of $\rho$ is bounded.

We will prove that for certain cases there are no stationary states to (1.2) in the sense of Definition 2.1, for instance for the sub-critical classical Keller-Segel model in two dimensions [9]. However, the scale invariance of (1.2) motivates us to look for self-similar solutions instead. To this end, we rescale equation (1.2) to a non-linear Fokker-Planck type equation as in [23]. Let us define

$$
u(t, x):=\alpha^{N}(t) \rho(\beta(t), \alpha(t) x),
$$

where $\rho(t, x)$ solves (1.2) and the functions $\alpha(t), \beta(t)$ are to be determined. If we assume $u(0, x)=\rho(0, x)$, then $u(t, x)$ satisfies the rescaled drift-diffusion equation

$$
\left\{\begin{array}{l}
\partial_{t} u=\frac{1}{N} \Delta u^{m}+2 \chi \nabla \cdot\left(u \nabla S_{k}\right)+\nabla \cdot(x u), \quad t>0, x \in \mathbb{R}^{N} \\
u(t=0, x)=\rho_{0}(x) \geq 0, \quad \int_{-\infty}^{\infty} \rho_{0}(x) d x=1, \quad \int_{-\infty}^{\infty} x \rho_{0}(x) d x=0
\end{array}\right.
$$

for the choices

$$
\alpha(t)=e^{t}, \quad \beta(t)= \begin{cases}\frac{1}{2-k}\left(e^{(2-k) t}-1\right), & \text { if } k \neq 2 \\ t, & \text { if } k=2\end{cases}
$$


and with $\nabla S_{k}$ given by (1.3) with $u$ instead of $\rho$. By differentiating the centre of mass of $u$, we see easily that

$$
\int_{\mathbb{R}^{N}} x u(t, x) d x=e^{-t} \int_{\mathbb{R}^{N}} x \rho_{0}(x) d x=0, \quad \forall t>0,
$$

and so the initial zero centre of mass is preserved for all times. Self-similar solutions to (1.2) now correspond to stationary solutions of (2.3). Similar to Definition 2.1, we state what we exactly mean by stationary states to the aggregation equation (2.3).

Definition 2.3. Given $\bar{u} \in L_{+}^{1}\left(\mathbb{R}^{N}\right) \cap L^{\infty}\left(\mathbb{R}^{N}\right)$ with $\|\bar{u}\|_{1}=1$, it is a stationary state for the evolution equation $(2.3)$ if $\bar{u}^{m} \in \mathcal{W}_{\text {loc }}^{1,2}\left(\mathbb{R}^{N}\right), \nabla \bar{S}_{k} \in L_{\text {loc }}^{1}\left(\mathbb{R}^{N}\right)$, and it satisfies

$$
\frac{1}{N} \nabla \bar{u}^{m}=-2 \chi \bar{u} \nabla \bar{S}_{k}-x \bar{u}
$$

in the sense of distributions in $\mathbb{R}^{N}$. If $-N<k \leq 1-N$, we further require $\bar{u} \in C^{0, \alpha}\left(\mathbb{R}^{N}\right)$ with $\alpha \in(1-k-N, 1)$.

From now on, we switch notation from $u$ to $\rho$ for simplicity, it should be clear from the context if we are in original or rescaled variables. In fact, stationary states as defined above have even more regularity:

Lemma 2.4. Let $k \in(-N, N) \backslash\{0\}$ and $\chi>0$.

(i) If $\bar{\rho}$ is a stationary state of Eq. (1.2) with $|x|^{k} \bar{\rho} \in L^{1}\left(\mathbb{R}^{N}\right)$ in the case $0<k<N$, then $\bar{\rho}$ is continuous on $\mathbb{R}^{N}$.

(ii) If $\bar{\rho}_{\text {resc }}$ is a stationary state of Eq. (2.3) with $|x|^{k} \bar{\rho}_{\text {resc }} \in L^{1}\left(\mathbb{R}^{N}\right)$ in the case $0<k<N$, then $\bar{\rho}_{\text {resc }}$ is continuous on $\mathbb{R}^{N}$.

Proof. (i) First, note that $\nabla \bar{S}_{k} \in L_{\text {loc }}^{\infty}\left(\mathbb{R}^{N}\right)$ by Lemma 2.2, and therefore, $\bar{\rho} \nabla \bar{S}_{k} \in L_{\text {loc }}^{1}\left(\mathbb{R}^{N}\right) \cap L_{\text {loc }}^{\infty}\left(\mathbb{R}^{N}\right)$. Hence, we get by interpolation that $\nabla \cdot\left(\bar{\rho} \nabla \bar{S}_{k}\right) \in \mathcal{W}_{l o c}^{-1, p}\left(\mathbb{R}^{N}\right)$ for all $1<p<\infty$. Recall from Definition 2.1 that $\bar{\rho}^{m}$ is a weak $\mathcal{W}_{\text {loc }}^{1,2}\left(\mathbb{R}^{N}\right)$ solution of

$$
\frac{1}{N} \Delta \bar{\rho}^{m}=-2 \chi \nabla \cdot\left(\bar{\rho} \nabla \bar{S}_{k}\right)
$$

in $\mathbb{R}^{N}$, and so $\bar{\rho}^{m}$ is in fact a weak solution in $\mathcal{W}_{\text {loc }}^{1, p}\left(\mathbb{R}^{N}\right)$ for all $1<p<\infty$ by classic elliptic regularity. Using Morrey's inequality, we deduce that $\bar{\rho}^{m}$ belongs to the Hölder space $C_{\text {loc }}^{0, \alpha}\left(\mathbb{R}^{N}\right)$ with $\alpha=(p-N) / N$ for any $N<p<\infty$, and thus $\bar{\rho}^{m} \in C\left(\mathbb{R}^{N}\right)$. Hence, $\bar{\rho}$ itself is continuous as claimed.

(ii) Since $x \bar{\rho}_{\text {resc }} \in L_{\text {loc }}^{1}\left(\mathbb{R}^{N}\right) \cap L_{\text {loc }}^{\infty}\left(\mathbb{R}^{N}\right)$, we obtain again by interpolation $\nabla \cdot\left(x \bar{\rho}_{\text {resc }}\right) \in \mathcal{W}_{\text {loc }}^{-1, p}\left(\mathbb{R}^{N}\right)$ for all $1<p<\infty$. By Definition 2.3, $\bar{\rho}_{\text {resc }}^{m}$ is a weak $\mathcal{W}_{\text {loc }}^{1,2}\left(\mathbb{R}^{N}\right)$ solution of

$$
\frac{1}{N} \Delta \bar{\rho}_{\text {resc }}^{m}=-2 \chi \nabla \cdot\left(\bar{\rho}_{\text {resc }} \nabla \bar{S}_{k}\right)-\nabla \cdot\left(x \bar{\rho}_{\text {resc }}\right)
$$

in $\mathbb{R}^{N}$, and so $\bar{\rho}_{\text {resc }}^{m}$ is again a weak solution in $\mathcal{W}_{\text {loc }}^{1, p}\left(\mathbb{R}^{N}\right)$ for all $1<p<\infty$ by classic elliptic regularity. We conclude as in original variables.

In the case $k<0$, we furthermore have a non-linear algebraic equation for stationary states:

Corollary 2.5 (Necessary Condition for Stationary States). Let $k \in(-N, 0)$ and $\chi>0$. 
(i) If $\bar{\rho}$ is a stationary state of Eq. (1.2), then $\bar{\rho} \in \mathcal{W}^{1, \infty}\left(\mathbb{R}^{N}\right)$ and it satisfies

$$
\bar{\rho}(x)^{m-1}=\frac{N(m-1)}{m}\left(C_{k}[\bar{\rho}](x)-2 \chi \bar{S}_{k}(x)\right)_{+}, \quad \forall x \in \mathbb{R}^{N}
$$

where $C_{k}[\bar{\rho}](x)$ is constant on each connected component of supp $(\bar{\rho})$.

(ii) If $\bar{\rho}_{\text {resc }}$ is a stationary state of $E q .(2.3)$, then $\bar{\rho}_{\text {resc }} \in \mathcal{W}_{\text {loc }}^{1, \infty}\left(\mathbb{R}^{N}\right)$ and it satisfies

$$
\bar{\rho}_{\text {resc }}(x)^{m-1}=\frac{N(m-1)}{m}\left(C_{k, \mathbf{r e s c}}[\bar{\rho}](x)-2 \chi \bar{S}_{k}(x)-\frac{|x|^{2}}{2}\right)_{+}, \quad \forall x \in \mathbb{R}^{N}
$$

where $C_{k, \mathbf{r e s c}}[\bar{\rho}](x)$ is constant on each connected component of $\operatorname{supp}\left(\bar{\rho}_{\text {resc }}\right)$.

Proof. (i) For a stationary state $\bar{\rho}$ of Eq. (1.2), let us define the set

$$
\Omega=\left\{x \in \mathbb{R}^{N}: \bar{\rho}(x)>0\right\}
$$

Since $\bar{\rho}$ is continuous by Lemma $2.4, \Omega$ is an open set with countably many open, possibly unbounded connected components. Let us take any bounded smooth connected open subset $\mathcal{U}$ such that $\overline{\mathcal{U}} \subset \Omega$. By continuity, $\bar{\rho}$ is bounded away from zero in $\mathcal{U}$, and thus $\nabla \bar{\rho}^{m-1}=\frac{m-1}{m \bar{\rho}} \nabla \bar{\rho}^{m}$ holds in the distributional sense in $\mathcal{U}$. From (2.1) in the definition of stationary states, we conclude that

$$
\nabla\left(\frac{m}{N(m-1)} \bar{\rho}^{m-1}+2 \chi \bar{S}_{k}\right)=0
$$

in the sense of distributions in $\Omega$. Hence, the function $C_{k}[\bar{\rho}](x):=\frac{m}{N(m-1)} \bar{\rho}^{m-1}(x)+2 \chi \bar{S}_{k}(x)$ is constant in each connected component of $\Omega$, and so (2.5) follows. Additionally, it follows from (2.7) that for any $x \in \mathbb{R}^{N}$

$$
\left|\nabla \bar{\rho}^{m-1}(x)\right|=\frac{2 \chi N(m-1)}{m}\left|\nabla \bar{S}_{k}(x)\right| \leq c
$$

for some constant $c>0$ since $\bar{S}_{k} \in \mathcal{W}^{1, \infty}\left(\mathbb{R}^{N}\right)$ by Lemma 2.2. Since $m \in(1,2)$, we conclude $\bar{\rho} \in \mathcal{W}^{1, \infty}\left(\mathbb{R}^{N}\right)$.

(ii) We follow the same argument for a stationary state $\bar{\rho}_{\text {resc }}$ of the rescaled equation $(2.3)$ and using (2.4) in Definition 2.3, we obtain

$$
\nabla\left(\frac{m}{N(m-1)} \bar{\rho}_{\text {resc }}^{m-1}+2 \chi \bar{S}_{k}+\frac{|x|^{2}}{2}\right)=0
$$

in the sense of distributions in $\Omega$. Here, the function $C_{k, \text { resc }}\left[\bar{\rho}_{\text {resc }}\right](x):=\frac{m}{N(m-1)} \bar{\rho}_{\text {resc }}^{m-1}(x)+2 \chi \bar{S}_{k}(x)+\frac{|x|^{2}}{2}$ is again constant in each connected component of supp $\left(\bar{\rho}_{\text {resc }}\right)$. Similarly, it follows from Lemma 2.2 that for any $\omega>0$ and $x \in B(0, \omega)$,

$$
\left|\nabla \bar{\rho}_{\text {resc }}^{m-1}(x)\right|=\frac{N(m-1)}{m}\left(2 \chi\left|\nabla \bar{S}_{k}(x)\right|+|x|\right) \leq c
$$

for some constant $c>0$, and so $\bar{\rho}_{\text {resc }} \in \mathcal{W}_{\text {loc }}^{1, \infty}\left(\mathbb{R}^{N}\right)$. 


\subsection{Fair-competition: main results}

It is worth noting that the functional $\mathcal{F}_{m, k}[\rho]$ possesses remarkable homogeneity properties. Indeed, the mass-preserving dilation $\rho_{\lambda}(x)=\lambda^{N} \rho(\lambda x)$ transforms the functionals as follows:

$$
\mathcal{U}_{m}\left[\rho_{\lambda}\right]= \begin{cases}\lambda^{N(m-1)} \mathcal{U}_{m}[\rho], & \text { if } m \neq 1 \\ \mathcal{U}_{m}[\rho]+\log \lambda, & \text { if } m=1\end{cases}
$$

and,

$$
\mathcal{W}_{k}\left[\rho_{\lambda}\right]= \begin{cases}\lambda^{-k} \mathcal{W}_{k}[\rho], & \text { if } k \neq 0 \\ \mathcal{W}_{k}[\rho]-\log \lambda, & \text { if } k=0\end{cases}
$$

This motivates the following classification:

\section{Definition 2.6 (Three Different Regimes).}

$N(m-1)+k=0$ : This is the fair-competition regime, where homogeneities of the two competing contributions exactly balance. If $k<0$, or equivalently $m>1$, then we will have a dichotomy according to $\chi>0$ (see Definition 2.7). Some variants of the HLS inequalities are very related to this dichotomy. This was already proven in [31,9] for the Keller-Segel case in $N=2$, and in [7] for the Keller-Segel case in $N \geq 3$. If $k>0$, that is $m<1$, no critical $\chi$ exists as we will prove in Section 4 .

$N(m-1)+k>0$ : This is the diffusion-dominated regime. Diffusion is strong, and is expected to overcome aggregation, whatever $\chi>0$ is. This domination effect means that solutions exist globally in time and are bounded uniformly in time [11,51,50]. Stationary states were found by minimisation of the free energy functional in two and three dimensions $[48,18]$ in the case of attractive Newtonian potentials. Stationary states are radially symmetric if $2-N \leq k<0$ as proven in [19]. Moreover, in the particular case of $N=2, k=0$, and $m>1$ it has been proved in [19] that the asymptotic behaviour is given by compactly supported stationary solutions independently of $\chi$.

$N(m-1)+k<0$ : This is the attraction-dominated regime. This regime is less understood. Self-attraction is strong, and can overcome the regularising effect of diffusion whatever $\chi>0$ is, but there also exist global in time regular solutions under some smallness assumptions, see [30,49,52,26,4,27,39]. Most of the results known up to date deal with attractive Newtonian interactions, that is $k=2-N$, in dimension $N \geq 3$. For a study with linear diffusion $m=1$ and $k<0$ in one dimension, see [14]. For the Newtonian case, global existence vs blow-up of weak solutions has been investigated for the diffusion coefficients $m=1$ [30], $1<m \leq 2-\frac{2}{N}$ [49,52], $m=\frac{2 N}{N+2}$ [26], $\frac{2 N}{N+2}<m<2-\frac{2}{N}[27,39]$ and for the whole range $0<m \leq 2-\frac{2}{N}$ [4]. It was shown in [30] for linear diffusion $m=1$ that global in time weak solutions exist for initial data with small enough $L^{N / 2}$-norm, whereas there are no global smooth solutions with fast decay if the second moment of the initial data is dominated by a power of the mass (with these two conditions being incompatible). For diffusion coefficient $m=\frac{2 N}{N+2}$ making the free energy functional conformal invariant, there exists a family of stationary solutions characterising the transition between blow-up and global existence of radially symmetric weak solutions [26]. The case $\frac{2 N}{N+2}<m<2-\frac{2}{N}$ has been studied in [27], where the authors suggest that the initial mass may not be an important quantity to classify existence vs blow-up of solutions with the behaviour depending on the free energy, the $L^{2 N /(N+2)}$-norm and the second moment of the initial data. In [39], the authors proved a uniform $L^{\infty}$-bound for weak solutions in the range where these stationary solutions exist given the initial data is uniformly bounded. As a consequence, uniqueness of weak solutions follows. We refer to [3] for a discussion with more general interaction potentials in the aggregation-dominated regime. 
We will here only concentrate on the fair-competition regime, and denote the corresponding energy functional by $\mathcal{F}_{k}[\rho]=\mathcal{F}_{1-k / N, k}[\rho]$. From now on, assume $N(m-1)+k=0$. Notice that the functional $\mathcal{F}_{k}$ is homogeneous in this regime, i.e.,

$$
\mathcal{F}_{k}\left[\rho_{\lambda}\right]=\lambda^{-k} \mathcal{F}_{k}[\rho] .
$$

The analysis in the fair-competition regime depends on the sign of $k$ :

Definition 2.7 (Three Different Cases in the Fair-competition Regime).

$k<0$ : This is the porous medium case with $m \in(1,2)$, where diffusion is small in regions of small densities. The classical porous medium equation, i.e. $\chi=0$, is very well studied, see [54] and the references therein. We have a dichotomy for existence of stationary states and global minimisers of the energy functional $\mathcal{F}_{k}$ depending on a critical parameter $\chi_{c}$ which will be defined in (3.5), and hence separate the sub-critical, the critical and the super-critical case, according to $\chi \lessgtr \chi_{c}$. The case $k<0$ is discussed in Section 3.

$k=0$ : This is the logarithmic case. There exists an explicit extremal density $\bar{\rho}_{0}$ which realises the minimum of the functional $\mathcal{F}_{0}$ when $\chi=1$. Moreover, the functional $\mathcal{F}_{0}$ is bounded below but does not achieve its infimum for $0<\chi<1$ while it is not bounded below for $\chi>1$. Hence, $\chi_{c}=1$ is the critical parameter in the logarithmic case that was analysed in [12] in one dimension and radial initial data in two dimensions.

$k>0$ : This is the fast diffusion case with $m \in(0,1)$, where diffusion is strong in regions of small densities. For any $\chi>0$, no radially symmetric non-increasing stationary states with bounded $k$ th moment exist, and $\mathcal{F}_{k}$ has no radially symmetric non-increasing minimisers. However, we have existence of self-similar profiles independently of $\chi>0$ as long as diffusion is not too fast, i.e. $k \leq 1$. Selfsimilar profiles can only exist if diffusion is not too strong with restriction $0<k<2$, that is $(N-2) / N<m<1$. The fast diffusion case is discussed in Section 4.

When dealing with the energy functional $\mathcal{F}_{k}$, we work in the set of non-negative normalised densities,

$$
\mathcal{Y}:=\left\{\rho \in L_{+}^{1}\left(\mathbb{R}^{N}\right) \cap L^{m}\left(\mathbb{R}^{N}\right):\|\rho\|_{1}=1, \int x \rho(x) d x=0\right\} .
$$

In rescaled variables, Eq. (2.3) is the formal gradient flow of the rescaled free energy functional $\mathcal{F}_{k, \text { resc }}$, which is complemented with an additional quadratic confinement potential,

$$
\mathcal{F}_{k, \mathbf{r e s c}}[\rho]=\mathcal{F}_{k}[\rho]+\frac{1}{2} \mathcal{V}[\rho], \quad \mathcal{V}[\rho]=\int_{\mathbb{R}^{N}}|x|^{2} \rho(x) d x
$$

Defining the sets

$$
\mathcal{Y}_{2}:=\{\rho \in \mathcal{Y}: \mathcal{V}[\rho]<\infty\}, \quad \mathcal{Y}_{k}:=\left\{\rho \in \mathcal{Y}: \int_{\mathbb{R}^{N}}|x|^{k} \rho(x) d x<\infty\right\},
$$

we see that $\mathcal{F}_{k \text {,resc }}$ is well-defined and finite on $\mathcal{Y}_{2}$ for $k<0$ and on $\mathcal{Y}_{2, k}:=\mathcal{Y}_{2} \cap \mathcal{Y}_{k}$ for $k>0$. Thanks to the formal gradient flow structure in the Euclidean Wasserstein metric $\mathbf{W}$, we can write the rescaled equation (2.3) as

$$
\partial_{t} \rho=\nabla \cdot\left(\rho \nabla \mathcal{T}_{k, \text { resc }}[\rho]\right)=-\nabla_{\mathbf{W}} \mathcal{F}_{k, \text { resc }}[\rho],
$$

where $\mathcal{T}_{k, \text { resc }}$ denotes the first variation of the rescaled energy functional,

$$
\mathcal{T}_{k, \operatorname{resc}}[\rho](x):=\mathcal{T}_{k}[\rho](x)+\frac{|x|^{2}}{2}
$$

with $\mathcal{T}_{k}$ as defined in (1.4). In this paper, we prove the following results: 
Theorem 2.8 (The Critical Porous Medium Regime). In the porous medium regime $k \in(-N, 0)$ and for critical interaction strengths $\chi=\chi_{c}$, there exist global minimisers of $\mathcal{F}_{k}$ and they are radially symmetric non-increasing, compactly supported and uniformly bounded. Furthermore, all stationary states with bounded second moment are global minimisers of the energy functional $\mathcal{F}_{k}$, and conversely, all global minimisers of $\mathcal{F}_{k}$ are stationary states of (1.2).

Theorem 2.9 (The Sub-Critical Porous Medium Regime). In the porous medium regime $k \in(-N, 0)$ and for sub-critical interaction strengths $0<\chi<\chi_{c}$, no stationary states exist for Eq. (1.2) and no minimisers exist for $\mathcal{F}_{k}$. In rescaled variables, all stationary states are continuous and compactly supported. There exist global minimisers of $\mathcal{F}_{k, \text { resc }}$ and they are radially symmetric non-increasing and uniformly bounded stationary states of Eq. (2.3).

Remark 2.10. Due to the homogeneity (2.8) of the functional $\mathcal{F}_{k}$, each global minimiser gives rise to a family of global minimisers for $\chi=\chi_{c}$ by dilation since they have zero energy, see (3.6). It is an open problem to show that there is a unique global minimiser for $\chi=\chi_{c}$ modulo dilations. This uniqueness was proven in the Newtonian case in [56]. We will explore the uniqueness modulo dilations of global minimisers in radial variables in a forthcoming paper. Notice that one obtains the full set of stationary states with bounded second moment for (1.2) as a by-product.

In contrast, in rescaled variables, we do not know if stationary states with second moment bounded are among global minimisers of $\mathcal{F}_{k, \text { resc }}$ for the sub-critical case $0<\chi<\chi_{c}$ except in one dimension, see [13]. It is also an open problem to show the uniqueness of radially symmetric stationary states of the rescaled equation (2.3) for $N \geq 2$.

Theorem 2.11 (The Fast Diffusion Regime). In the fast diffusion regime $k \in(0, N)$ Eq. (1.2) has no radially symmetric non-increasing stationary states with $k$ th moment bounded, and there are no radially symmetric non-increasing global minimisers for the energy functional $\mathcal{F}_{k}$ for any $\chi>0$. In rescaled variables, radially symmetric non-increasing stationary states can only exist if $0<k<2$, that is $(N-2) / N<m<1$. Similarly, global minimisers with finite energy $\mathcal{F}_{k, \mathbf{r e s c}}$ can only exist in the range $0<k<2 N /(2+N)$, that is $N /(2+N)<m<1$. For $k \in(0,1]$, there exists a continuous radially symmetric non-increasing stationary state of the rescaled equation (2.3).

\section{Porous medium case $k<0$}

In the porous medium case, we have $-N<k<0$ and hence $1<m<2$. Our aim in this section is to make a connection between global minimisers of the functionals $\mathcal{F}_{k}$ and $\mathcal{F}_{k \text {,resc }}$ and stationary states of Eqs. (1.2) and (2.3) respectively. We will show that in the critical case $\chi=\chi_{c}$, global minimisers and stationary states are equivalent for original variables. In the sub-critical case $0<\chi<\chi_{c}$, all minimisers of $\mathcal{F}_{k, \text { resc }}$ will turn out to be stationary states of the rescaled equation (2.3).

\subsection{Global minimisers}

A key ingredient for the analysis in the porous medium case is certain functional inequalities which are variants of the Hardy-Littlewood-Sobolev (HLS) inequality, also known as the weak Young's inequality 
[38, Theorem 4.3]:

$$
\begin{aligned}
& \iint_{\mathbb{R}^{N} \times \mathbb{R}^{N}} f(x)|x-y|^{k} f(y) d x d y \leq C_{H L S}(p, q, k)\|f\|_{p}\|f\|_{q} \\
& \frac{1}{p}+\frac{1}{q}=2+\frac{k}{N}, \quad p, q>1, k \in(-N, 0) .
\end{aligned}
$$

Theorem 3.1 (Variation of $H L S)$. Let $k \in(-N, 0)$. For $f \in L^{1}\left(\mathbb{R}^{N}\right) \cap L^{m}\left(\mathbb{R}^{N}\right)$, we have

$$
\left|\iint_{\mathbb{R}^{N} \times \mathbb{R}^{N}} f(x)\right| x-\left.y\right|^{k} f(y) d x d y \mid \leq C_{*}\|f\|_{1}^{\frac{N+k}{N}}\|f\|_{m}^{m}
$$

where $C_{*}(k, N)$ is defined as the best constant.

Proof. The inequality is a direct consequence of the standard HLS inequality (3.1) by choosing $p=q=\frac{2 N}{2 N+k}$, and of Hölder's inequality. For $k \in(-N, 0)$ and for any $f \in L^{1}\left(\mathbb{R}^{N}\right) \cap L^{m}\left(\mathbb{R}^{N}\right)$, we have

$$
\left|\iint_{\mathbb{R} \times \mathbb{R}} f(x)\right| x-\left.y\right|^{k} f(y) d x d y \mid \leq C_{H L S}\|f\|_{p}^{2} \leq C_{H L S}\|f\|_{1}^{\frac{N+k}{N}}\|f\|_{m}^{m} .
$$

Consequently, $C_{*}$ is finite and bounded from above by $C_{H L S}$.

Now, let us compute explicitly the energy of stationary states:

Lemma 3.2. For any $-N<k<0$ and $\chi>0$, all stationary states $\bar{\rho}$ of $(1.2)$ with $|x|^{2} \bar{\rho} \in L^{1}\left(\mathbb{R}^{N}\right)$ satisfy $\mathcal{F}_{k}[\bar{\rho}]=0$.

Proof. Integrating (2.1) against $x$, we obtain for $1-N<k<0$ :

$$
\begin{aligned}
& \frac{1}{N} \int_{\mathbb{R}^{N}} x \cdot \nabla \bar{\rho}^{m}=-2 \chi \iint_{\mathbb{R}^{N} \times \mathbb{R}^{N}} x \cdot(x-y)|x-y|^{k-2} \bar{\rho}(x) \bar{\rho}(y) d x d y \\
& \quad-\int_{\mathbb{R}^{N}} \bar{\rho}^{m}=-\chi \iint_{\mathbb{R}^{N} \times \mathbb{R}^{N}}(x-y) \cdot(x-y)|x-y|^{k-2} \bar{\rho}(x) \bar{\rho}(y) d x d y \\
& \frac{1}{N(m-1)} \int_{\mathbb{R}^{N}} \bar{\rho}^{m}=-\chi \iint_{\mathbb{R}^{N} \times \mathbb{R}^{N}} \frac{|x-y|^{k}}{k} \bar{\rho}(x) \bar{\rho}(y) d x d y,
\end{aligned}
$$

and the result immediately follows. For $-N<k \leq 1-N$, the term $\nabla \bar{S}_{k}$ is a singular integral, and thus writes

$$
\begin{aligned}
\nabla \bar{S}_{k}(x) & =\lim _{\varepsilon \rightarrow 0} \int_{B^{c}(x, \varepsilon)}|x-y|^{k-2}(x-y) \bar{\rho}_{k}(y) d y \\
& =\int_{\mathbb{R}^{N}}|x-y|^{k-2}(x-y)\left(\bar{\rho}_{k}(y)-\bar{\rho}_{k}(x)\right) d y .
\end{aligned}
$$

The singularity disappears when integrating against $x$,

$$
\int_{\mathbb{R}^{N}} x \cdot \nabla \bar{S}_{k}(x) \bar{\rho}_{k}(x) d x=\frac{1}{2} \iint_{\mathbb{R}^{N} \times \mathbb{R}^{N}}|x-y|^{k} \bar{\rho}_{k}(x) \bar{\rho}_{k}(y) d x d y .
$$

In order to prove (3.4), let us define $f_{\varepsilon}: \mathbb{R}^{N} \rightarrow \mathbb{R}$ for any $\varepsilon>0$ by

$$
f_{\varepsilon}(x):=\int_{B^{c}(x, \varepsilon)} x \cdot \nabla_{x} W_{k}(x-y) \bar{\rho}_{k}(y) d y .
$$

Then by definition of the Cauchy principle value, $f_{\varepsilon}(x)$ converges to $x \cdot \nabla \bar{S}_{k}(x)$ pointwise for almost every $x \in \mathbb{R}^{N}$ as $\varepsilon \rightarrow 0$. Further, we use the fact that $\bar{\rho}_{k} \in C^{0, \alpha}\left(\mathbb{R}^{N}\right)$ for some $\alpha \in(1-k-N, 1)$ to obtain the 
uniform in $\varepsilon$ estimate

$$
\left|f_{\varepsilon}(x)\right| \leq\left(\frac{\sigma_{N}+k+N-1+\alpha}{k+N-1+\alpha}\right)|x|, \quad \forall 0<\varepsilon<1,
$$

and therefore by Lebesgue's dominated convergence theorem,

$$
\begin{aligned}
\int_{\mathbb{R}^{N}} x \cdot \nabla \bar{S}_{k}(x) \bar{\rho}_{k}(x) d x & =\int_{\mathbb{R}^{N}} \lim _{\varepsilon \rightarrow 0} f_{\varepsilon}(x) \bar{\rho}_{k}(x) d x=\lim _{\varepsilon \rightarrow 0} \int_{\mathbb{R}^{N}} f_{\varepsilon}(x) \bar{\rho}_{k}(x) d x \\
& =\lim _{\varepsilon \rightarrow 0} \iint_{|x-y| \geq \varepsilon} x \cdot(x-y)|x-y|^{k-2} \bar{\rho}_{k}(x) \bar{\rho}_{k}(y) d x d y \\
& =\frac{1}{2} \lim _{\varepsilon \rightarrow 0} \iint_{|x-y| \geq \varepsilon}(x-y) \cdot(x-y)|x-y|^{k-2} \bar{\rho}_{k}(x) \bar{\rho}_{k}(y) d x d y \\
& =\frac{1}{2} \iint_{\mathbb{R}^{N} \times \mathbb{R}^{N}}|x-y|^{k} \bar{\rho}_{k}(x) \bar{\rho}_{k}(y) d x d y .
\end{aligned}
$$

This concludes the proof of (3.4).

Therefore, it follows that (3.3) holds true for any $-N<k<0$. We remark that a bounded second moment is necessary to allow for the use of $|x|^{2} / 2$ as a test function by a standard approximation argument using suitable truncations.

Let us point out that the previous computation is possible due to the homogeneity of the functional $\mathcal{F}_{k}$. In fact, a formal use of the Euler theorem for homogeneous functions leads to this statement. This argument does not apply in the logarithmic case $k=0$. Here, it allows to connect stationary states and minimisers of $\mathcal{F}_{k}$.

It follows directly from Theorem 3.1, that for all $\rho \in \mathcal{Y}$ and for any $\chi>0$,

$$
\mathcal{F}_{k}[\rho] \geq \frac{1-\chi C_{*}}{N(m-1)}\|\rho\|_{m}^{m}
$$

where $C_{*}=C_{*}(k, N)$ is the optimal constant defined in (3.2). Since global minimisers have always smaller or equal energy than stationary states, and stationary states have zero energy by Lemma 3.2, it follows that $\chi \geq 1 / C_{*}$. We define the critical interaction strength by

$$
\chi_{c}(k, N):=\frac{1}{C_{*}(k, N)},
$$

and so for $\chi=\chi_{c}$, all stationary states of Eq. (1.2) are global minimisers of $\mathcal{F}_{k}$. We can also directly see that for $0<\chi<\chi_{c}$, no stationary states exist. These observations can be summarised in the following theorem:

Theorem 3.3 (Stationary States in Original Variables). Let $-N<k<0$. For critical interaction strength $\chi=\chi_{c}$, all stationary states $\bar{\rho}$ of $E q .(1.2)$ with $|x|^{2} \bar{\rho} \in L^{1}\left(\mathbb{R}^{N}\right)$ are global minimisers of $\mathcal{F}_{k}$. For sub-critical interaction strengths $0<\chi<\chi_{c}$, no stationary states with $|x|^{2} \bar{\rho} \in L^{1}\left(\mathbb{R}^{N}\right)$ exist for $E q$. (1.2).

We now turn to the study of global minimisers of $\mathcal{F}_{k}$ and $\mathcal{F}_{k \text {,resc }}$ with the aim of proving the converse implication to Theorem 3.3. Firstly, we have the following existence result:

Proposition 3.4 (Existence of Global Minimisers). Let $k \in(-N, 0)$.

(i) If $\chi=\chi_{c}$, then there exists a radially symmetric and non-increasing function $\tilde{\rho} \in \mathcal{Y}$ satisfying $\mathcal{F}_{k}[\tilde{\rho}]=0$.

(ii) If $\chi<\chi_{c}$, then $\mathcal{F}_{k}$ does not admit global minimisers, but there exists a global minimiser $\tilde{\rho}$ of $\mathcal{F}_{k \text {,resc }}$ in $\mathcal{Y}_{2}$.

(iii) If $\chi>\chi_{c}$, then both $\mathcal{F}_{k}$ and $\mathcal{F}_{k \text {,resc }}$ are not bounded below. 
Proof. Generalising the argument in [7, Proposition 3.4], we obtain the following result for the behaviour of the free energy functional $\mathcal{F}_{k}$ : Let $\chi>0$. For $k \in(-N, 0)$, we have

$$
I_{k}(\chi):=\inf _{\rho \in \mathcal{Y}} \mathcal{F}_{k}[\rho]= \begin{cases}0 & \text { if } \chi \in\left(0, \chi_{c}\right], \\ -\infty & \text { if } \chi>\chi_{c},\end{cases}
$$

and the infimum $I_{k}(\chi)$ is only achieved if $\chi=\chi_{c}$. This implies statements (ii) and (iii) for $\mathcal{F}_{k}$. Case (iii) directly follows also in rescaled variables as in [7, Proposition 5.1]. The argument in the sub-critical case (ii) for $\mathcal{F}_{k, \text { resc }}$ is a bit more subtle than in the critical case (i) since we need to make sure that the second moment of our global minimiser is bounded. We will here only prove (ii) for rescaled variables, as (i) and (ii) in original variables are straightforward generalisations from [7, Lemma 3.3] and [7, Proposition 3.4] respectively.

Inequality (3.2) implies that the rescaled free energy is bounded on $\mathcal{Y}_{2}$ by

$$
-\frac{C_{*}}{k}\left(\chi_{c}+\chi\right)\|\rho\|_{m}^{m}+\frac{1}{2} \mathcal{V}[\rho] \geq \mathcal{F}_{k, \mathbf{r e s c}}[\rho] \geq-\frac{C_{*}}{k}\left(\chi_{c}-\chi\right)\|\rho\|_{m}^{m}+\frac{1}{2} \mathcal{V}[\rho],
$$

and it follows that the infimum of $\mathcal{F}_{k, \text { resc }}$ over $\mathcal{Y}_{2}$ in the sub-critical case is non negative. Hence, there exists a minimising sequence $\left(p_{j}\right) \in \mathcal{Y}_{2}$,

$$
\mathcal{F}_{k, \text { resc }}\left[p_{j}\right] \rightarrow \mu:=\inf _{\rho \in \mathcal{Y}_{2}} \mathcal{F}_{k, \text { resc }}[\rho] .
$$

Note that $\left\|p_{j}\right\|_{m}$ and $\mathcal{V}\left[p_{j}\right]$ are uniformly bounded, $\left\|p_{j}\right\|_{m}+\mathcal{V}\left[p_{j}\right] \leq C_{0}$ say, since from (3.7)

$$
0<-\frac{C_{*}}{k}\left(\chi_{c}-\chi\right)\left\|p_{j}\right\|_{m}^{m}+\frac{1}{2} \mathcal{V}\left[p_{j}\right] \leq \mathcal{F}_{k, \mathbf{r e s c}}\left[p_{j}\right] \leq \mathcal{F}_{k, \mathbf{r e s c}}\left[p_{0}\right] .
$$

Further, the radially symmetric decreasing rearrangement $\left(p_{j}^{*}\right)$ of $\left(p_{j}\right)$ satisfies

$$
\left\|p_{j}^{*}\right\|_{m}=\left\|p_{j}\right\|_{m}, \quad \mathcal{V}\left[p_{j}^{*}\right] \leq \mathcal{V}\left[p_{j}\right], \quad \mathcal{W}_{k}\left[p_{j}^{*}\right] \leq \mathcal{W}_{k}\left[p_{j}\right]
$$

by the reversed Hardy-Littlewood-Sobolev inequality [37] and Riesz rearrangement inequality [38]. In other words, $\mathcal{F}_{k, \text { resc }}\left[p_{j}^{*}\right] \leq \mathcal{F}_{k, \text { resc }}\left[p_{j}\right]$ and so $\left(p_{j}^{*}\right)$ is also a minimising sequence.

To show that the infimum is achieved, we start by showing that $\left(p_{j}^{*}\right)$ is uniformly bounded at a point. For any choice of $R>0$, we have

$$
\begin{aligned}
1=\left\|p_{j}^{*}\right\|_{1} & =\sigma_{N} \int_{0}^{\infty} p_{j}^{*}(r) r^{N-1} d r \\
& \geq \sigma_{N} \int_{0}^{R} p_{j}^{*}(r) r^{N-1} d r \geq \sigma_{N} \frac{R^{N}}{N} p_{j}^{*}(R) .
\end{aligned}
$$

Similarly, since $\left\|p_{j}^{*}\right\|_{m}$ is uniformly bounded,

$$
\begin{aligned}
C_{0} & \geq\left\|p_{j}^{*}\right\|_{m}^{m}=\sigma_{N} \int_{0}^{\infty} r^{N-1} p_{j}^{*}(r)^{m} d r \\
& \geq \sigma_{N} \int_{0}^{R} r^{N-1} p_{j}^{*}(r)^{m} d r \geq \sigma_{N} \frac{R^{N}}{N} p_{j}^{*}(R)^{m} .
\end{aligned}
$$

We conclude that

$$
0 \leq p_{j}(R) \leq b(R):=C_{1} \inf \left\{R^{-N}, R^{-\frac{N}{m}}\right\}, \quad \forall R>0
$$

for a positive constant $C_{1}$ only depending on $N, m$ and $C_{0}$. Then by Helly's Selection Theorem there exists a subsequence $\left(p_{j_{n}}^{*}\right)$ and a non-negative function $\tilde{\rho}: \mathbb{R}^{N} \rightarrow \mathbb{R}$ such that $p_{j_{n}}^{*} \rightarrow \tilde{\rho}$ pointwise almost everywhere. In addition, a direct calculation shows that $x \mapsto b(|x|) \in L^{\frac{2 N}{2 N+k}}\left(\mathbb{R}^{N}\right)$, and hence, using (3.1) 
for $p=q=2 N /(2 N+k)$, we obtain

$$
(x, y) \mapsto|x-y|^{k} b(|x|) b(|y|) \in L^{1}\left(\mathbb{R}^{N} \times \mathbb{R}^{N}\right) .
$$

Together with (3.8) and the pointwise convergence of $\left(p_{j_{n}}^{*}\right)$, we conclude

$$
\mathcal{W}_{k}\left(p_{j_{n}}^{*}\right) \rightarrow \mathcal{W}_{k}(\tilde{\rho})<\infty
$$

by Lebesgue's dominated convergence theorem. In fact, since $\left\|p_{j_{n}}^{*}\right\|_{m}$ and $\mathcal{V}\left[p_{j_{n}}^{*}\right]$ are uniformly bounded and $\left\|p_{j_{n}}^{*}\right\|_{1}=1$, we have the existence of a subsequence $\left(p_{j_{l}}^{*}\right)$ and a limit $P \in L^{1}\left(\mathbb{R}^{N}\right)$ such that $p_{j_{l}}^{*} \rightarrow P$ weakly in $L^{1}\left(\mathbb{R}^{N}\right)$ by the Dunford-Pettis Theorem. Using a variant of Vitali's Lemma [44], we see that the sequence $\left(p_{j_{l}}^{*}\right)$ actually converges strongly to $\tilde{\rho}$ in $L^{1}\left(\mathbb{R}^{N}\right)$ on all finite balls in $\mathbb{R}^{N}$. In other words, $P=\tilde{\rho}$ almost everywhere. Furthermore, $\tilde{\rho}$ has finite second moment by Fatou's Lemma,

$$
\mathcal{V}[\tilde{\rho}] \leq \liminf _{l \rightarrow \infty} \mathcal{V}\left[p_{j_{l}}^{*}\right] \leq C_{0},
$$

and by convexity of $|\cdot|^{m}$ for $m \in(1,2)$, we have lower semi-continuity,

$$
\int \tilde{\rho}^{m} \leq \liminf _{l \rightarrow \infty} \int\left(p_{j_{l}}^{*}\right)^{m} \leq C_{0} .
$$

We conclude that $\tilde{\rho} \in \mathcal{Y}_{2}$ and

$$
\mathcal{F}_{k, \text { resc }}[\tilde{\rho}] \leq \lim _{l \rightarrow \infty} \mathcal{F}_{k, \text { resc }}\left[p_{j_{l}}^{*}\right]=\mu .
$$

Hence, $\tilde{\rho}$ is a global minimiser of $\mathcal{F}_{k, \text { resc }}$.

Remark 3.5. The existence result in original variables also provides optimisers for the variation of the HLS inequality (3.2), and so the supremum in the definition of $C_{*}(N, k)$ is in fact attained.

The following necessary condition is a generalisation of results in [7], but using a different argument inspired by [18].

Proposition 3.6 (Necessary Condition for Global Minimisers). Let $k \in(-N, 0)$.

(i) If $\chi=\chi_{c}$ and $\rho \in \mathcal{Y}$ is a global minimiser of $\mathcal{F}_{k}$, then $\rho$ is radially symmetric non-increasing, satisfying

$$
\rho^{m-1}(x)=\frac{N(m-1)}{m}\left(-2 \chi\left(W_{k} * \rho\right)(x)+D_{k}[\rho]\right)_{+} \quad \text { a.e. in } \mathbb{R}^{N} .
$$

Here, we denote

$$
D_{k}[\rho]:=2 \mathcal{F}_{k}[\rho]+\frac{m-2}{N(m-1)}\|\rho\|_{m}^{m}
$$

(ii) If $0<\chi<\chi_{c}$ and $\rho \in \mathcal{Y}_{2}$ is a global minimiser of $\mathcal{F}_{k \text {,resc }}$, then $\rho$ is radially symmetric non-increasing, satisfying

$$
\rho^{m-1}(x)=\frac{N(m-1)}{m}\left(-2 \chi\left(W_{k} * \rho\right)(x)-\frac{|x|^{2}}{2}+D_{k, \mathbf{r e s c}}[\rho]\right)_{+} \quad \text { a.e. in } \mathbb{R}^{N} .
$$

Here, we denote

$$
D_{k, \mathbf{r e s c}}[\rho]:=2 \mathcal{F}_{k, \mathbf{r e s c}}[\rho]-\frac{1}{2} \mathcal{V}[\rho]+\frac{m-2}{N(m-1)}\|\rho\|_{m}^{m}
$$


Proof. (i) Let us write as in (1.1)

$$
\begin{aligned}
& \mathcal{F}_{k}[\rho]=\mathcal{U}_{1-k / N}[\rho]+\chi \mathcal{W}_{k}[\rho], \quad \mathcal{U}_{m}[\rho]=\frac{1}{N(m-1)}\|\rho\|_{m}^{m}, \quad \text { and } \\
& \mathcal{W}_{k}[\rho]=\iint_{\mathbb{R}^{N} \times \mathbb{R}^{N}} \frac{|x-y|^{k}}{k} \rho(x) \rho(y) d x d y .
\end{aligned}
$$

We will first show that all global minimisers of $\mathcal{F}_{k}$ are radially symmetric non-increasing. Indeed, let $\rho$ be a global minimiser of $\mathcal{F}_{k}$ in $\mathcal{Y}$, then for the symmetric decreasing rearrangement $\rho^{*}$ of $\rho$, we have $\mathcal{U}_{m}\left[\rho^{*}\right]=\mathcal{U}_{m}[\rho]$ and by the Riesz rearrangement inequality [17, Lemma 2$], \mathcal{W}\left[\rho^{*}\right] \leq \mathcal{W}[\rho]$. So $\mathcal{F}_{k}\left[\rho^{*}\right] \leq \mathcal{F}_{k}[\rho]$ and since $\rho$ is a global minimiser this implies $\mathcal{W}_{k}\left[\rho^{*}\right]=\mathcal{W}_{k}[\rho]$. By Riesz rearrangement properties [17, Lemma 2], there exists $x_{0} \in \mathbb{R}^{N}$ such that $\rho(x)=\rho^{*}\left(x-x_{0}\right)$ for all $x \in \mathbb{R}^{N}$. Moreover, we have

$$
\int_{\mathbb{R}^{N}} x \rho(x) d x=x_{0}+\int_{\mathbb{R}^{N}} x \rho^{*}(x) d x=x_{0},
$$

and thus the zero centre-of-mass condition holds if and only if $x_{0}=0$, giving $\rho=\rho^{*}$. For any test function $\psi \in C_{c}^{\infty}\left(\mathbb{R}^{N}\right)$ such that $\psi(-x)=\psi(x)$, we define

$$
\varphi(x)=\rho(x)\left(\psi(x)-\int_{\mathbb{R}^{N}} \psi(x) \rho(x) d x\right) .
$$

We fix $0<\varepsilon<\varepsilon_{0}:=\left(2\|\psi\|_{\infty}\right)^{-1}$. Then

$$
\rho+\varepsilon \varphi=\rho\left(1+\varepsilon\left(\psi-\int_{\mathbb{R}^{N}} \psi \rho\right)\right) \geq \rho\left(1-2\|\psi\|_{\infty} \varepsilon\right) \geq 0
$$

and so $\rho+\varepsilon \varphi \in L_{+}^{1}\left(\mathbb{R}^{N}\right) \cap L^{m}\left(\mathbb{R}^{N}\right)$. Further, $\int \varphi(x) d x=\int x \varphi(x) d x=0$, and hence $\rho+\varepsilon \varphi \in \mathcal{Y}$. Note also that $\operatorname{supp}(\varphi) \subseteq \bar{\Omega}:=\operatorname{supp}(\rho)$. To calculate the first variation $\mathcal{T}_{k}$ of the functional $\mathcal{F}_{k}$, we need to be careful about regularity issues. Denoting by $\Omega$ the interior of $\bar{\Omega}$, we write

$$
\begin{aligned}
\frac{\mathcal{F}_{k}[\rho+\varepsilon \varphi]-\mathcal{F}_{k}[\rho]}{\varepsilon} & =\frac{1}{N(m-1)} \int_{\Omega} \frac{(\rho+\varepsilon \varphi)^{m}-\rho^{m}}{\varepsilon} d x+2 \chi \int_{\mathbb{R}^{N}}\left(W_{k} * \rho\right)(x) \varphi(x) d x+\varepsilon \mathcal{W}_{k}[\varphi] \\
& =\frac{m}{N(m-1)} \int_{0}^{1} \mathcal{G}_{\varepsilon}(t) d t+2 \chi \int_{\mathbb{R}^{N}}\left(W_{k} * \rho\right)(x) \varphi(x) d x+\varepsilon \mathcal{W}_{k}[\varphi],
\end{aligned}
$$

where $\mathcal{G}_{\varepsilon}(t):=\int_{\Omega}|\rho+t \varepsilon \varphi|^{m-2}(\rho+t \varepsilon \varphi) \varphi d x$. Then by Hölder's inequality,

$$
\left|\mathcal{G}_{\varepsilon}(t)\right| \leq\left(\|\rho\|_{m}+\varepsilon_{0}\|\varphi\|_{m}\right)^{m-1}\|\varphi\|_{m}
$$

for all $t \in[0,1]$ and $\varepsilon \in\left(0, \varepsilon_{0}\right)$. Lebesgue's dominated convergence theorem yields

$$
\int_{0}^{1} \mathcal{G}_{\varepsilon}(t) d t \rightarrow \int_{\Omega} \rho^{m-1}(x) \varphi(x) d x
$$


as $\varepsilon \rightarrow 0$. In addition, one can verify that $\mathcal{W}_{k}[\varphi] \leq 4\|\psi\|_{\infty}^{2} \mathcal{W}_{k}[\rho]<\infty$. Hence,

$$
\begin{aligned}
\lim _{\varepsilon \rightarrow 0}\left(\frac{\mathcal{F}_{k}[\rho+\varepsilon \varphi]-\mathcal{F}_{k}[\rho]}{\varepsilon}\right) & =\frac{m}{N(m-1)} \int_{\Omega} \rho^{m-1}(x) \varphi(x) d x+2 \chi \int_{\mathbb{R}^{N}}\left(W_{k} * \rho\right)(x) \varphi(x) d x \\
& =\int_{\mathbb{R}^{N}} \mathcal{T}_{k}[\rho](x) \varphi(x) d x,
\end{aligned}
$$

proving (1.4). Since $\rho$ is a global minimiser, $\mathcal{F}_{k}[\rho+\varepsilon \varphi] \geq \mathcal{F}_{k}[\rho]$ and hence $\int \mathcal{T}_{k}[\rho](x) \varphi(x) d x \geq 0$. Taking $-\psi$ instead of $\psi$, we obtain by the same argument $\int \mathcal{T}_{k}[\rho](x) \varphi(x) d x \leq 0$, and so

$$
\int_{\mathbb{R}^{N}} \mathcal{T}_{k}[\rho](x) \varphi(x) d x=0
$$

Owing to the choice of $\varphi$,

$$
\begin{aligned}
0 & =\int_{\mathbb{R}^{N}} \mathcal{T}_{k}[\rho](x) \varphi(x) d x \\
& =\int_{\mathbb{R}^{N}} \mathcal{T}_{k}[\rho](x) \rho(x) \psi(x) d x-\left(\int_{\mathbb{R}^{N}} \psi \rho\right)\left(2 \mathcal{F}_{k}[\rho]+\frac{m-2}{N(m-1)}\|\rho\|_{m}^{m}\right) \\
& =\int_{\mathbb{R}^{N}} \rho(x) \psi(x)\left(\mathcal{T}_{k}[\rho](x)-D_{k}[\rho]\right) d x
\end{aligned}
$$

for any symmetric test function $\psi \in C_{c}^{\infty}\left(\mathbb{R}^{N}\right)$. Hence $\mathcal{T}_{k}[\rho](x)=D_{k}[\rho]$ a.e. in $\bar{\Omega}$, i.e.

$$
\rho^{m-1}(x)=\frac{N(m-1)}{m}\left(-2 \chi\left(W_{k} * \rho\right)(x)+D_{k}[\rho]\right) \quad \text { a.e. in } \bar{\Omega} .
$$

Now, we turn to conditions over $\rho$ on the whole space. Let $\psi \in C_{c}^{\infty}\left(\mathbb{R}^{N}\right), \psi(-x)=\psi(x), \psi \geq 0$, and define

$$
\varphi(x):=\psi(x)-\rho(x) \int_{\mathbb{R}^{N}} \psi(x) d x \quad \in L^{1}\left(\mathbb{R}^{N}\right) \cap L^{m}\left(\mathbb{R}^{N}\right) .
$$

Then for $0<\varepsilon<\varepsilon_{0}:=\left(\|\psi\|_{\infty}|\operatorname{supp}(\psi)|\right)^{-1}$, we have

$$
\rho+\varepsilon \varphi \geq \rho\left(1-\varepsilon \int_{\mathbb{R}^{N}} \psi\right) \geq \rho\left(1-\varepsilon\|\psi\|_{\infty}|\operatorname{supp}(\psi)|\right)
$$

So $\rho+\varepsilon \varphi \geq 0$ in $\bar{\Omega}$, and also outside $\bar{\Omega}$ since $\psi \geq 0$, hence $\rho+\varepsilon \varphi \in \mathcal{Y}$. Repeating the previous argument, we obtain

$$
\int_{\mathbb{R}^{N}} \mathcal{T}_{k}[\rho](x) \varphi(x) d x \geq 0
$$

Using the expression of $\varphi$, we have

$$
\begin{aligned}
0 & \leq \int_{\mathbb{R}^{N}} \mathcal{T}_{k}[\rho](x) \varphi(x) d x \\
& =\int_{\mathbb{R}^{N}} \mathcal{T}_{k}[\rho](x) \psi(x) d x-\left(\int_{\mathbb{R}^{N}} \psi\right)\left(2 \mathcal{F}_{k}[\rho]+\frac{m-2}{N(m-1)}\|\rho\|_{m}^{m}\right) \\
& =\int_{\mathbb{R}^{N}} \psi(x)\left(\mathcal{T}_{k}[\rho](x)-D_{k}[\rho]\right) d x .
\end{aligned}
$$


Hence $\mathcal{T}_{k}[\rho](x) \geq D_{k}[\rho]$ a.e. in $\mathbb{R}^{N}$, and so

$$
\rho^{m-1}(x) \geq \frac{N(m-1)}{m}\left(-2 \chi\left(W_{k} * \rho\right)(x)+D_{k}[\rho]\right) \quad \text { a.e. in } \mathbb{R}^{N}
$$

Note that (3.12) means that the support $\bar{\Omega}$ coincides with the set

$$
\left\{x \in \mathbb{R}^{N} \mid-2 \chi\left(W_{k} * \rho\right)(x)+D_{k}[\rho]>0\right\} .
$$

Combining (3.11) and (3.12) completes the proof of (3.9).

(ii) First, note that if $\rho \in \mathcal{Y}_{2}$ and $\rho^{*}$ denotes the symmetric decreasing rearrangement of $\rho$, then it follows from the reversed Hardy-Littlewood-Sobolev inequality [37] that $\mathcal{V}\left[\rho^{*}\right] \leq \mathcal{V}[\rho]$. Since $\mathcal{U}_{m}\left[\rho^{*}\right]=\mathcal{U}_{m}[\rho]$ and $\mathcal{W}\left[\rho^{*}\right] \leq \mathcal{W}[\rho]$, we conclude $\mathcal{F}_{k, \text { resc }}\left[\rho^{*}\right] \leq \mathcal{F}_{k, \text { resc }}[\rho]$. For a global minimiser $\rho \in \mathcal{Y}_{2}$, we have $\mathcal{F}_{k, \text { resc }}\left[\rho^{*}\right]=\mathcal{F}_{k, \text { resc }}[\rho]$ and hence $\mathcal{W}\left[\rho^{*}\right]=\mathcal{W}[\rho]$ and $\mathcal{V}\left[\rho^{*}\right]=\mathcal{V}[\rho]$. The former implies that there exists $x_{0} \in \mathbb{R}^{N}$ such that $\rho(x)=\rho^{*}\left(x-x_{0}\right)$ for all $x \in \mathbb{R}^{N}$ by Riesz rearrangement properties [17, Lemma 2], and so the equality in second moment gives $\rho=\rho^{*}$.

Next, we will derive Eq. (3.10). We define for any test function $\psi \in C_{c}^{\infty}\left(\mathbb{R}^{N}\right)$ the function $\varphi(x)=\rho(x)\left(\psi(x)-\int_{\mathbb{R}^{N}} \psi(x) \rho(x) d x\right)$, and by the same argument as in (i), we obtain

$$
0=\int_{\mathbb{R}^{N}} \mathcal{T}_{k, \mathbf{r e s c}}[\rho](x) \varphi(x) d x=\int_{\mathbb{R}^{N}} \rho(x) \psi(x)\left(\mathcal{T}_{k, \mathbf{r e s c}}[\rho](x)-D_{k, \mathbf{r e s c}}[\rho]\right) d x,
$$

with $\mathcal{T}_{k, \text { resc }}$ as given in $(2.9)$. Hence $\mathcal{T}_{k, \mathbf{r e s c}}[\rho](x)=D_{k, \mathbf{r e s c}}[\rho]$ a.e. in $\bar{\Omega}:=\operatorname{supp}(\rho)$. Following the same argument as in (i), we further conclude $\mathcal{T}_{k, \text { resc }}[\rho](x) \geq D_{k, \text { resc }}[\rho]$ a.e. in $\mathbb{R}^{N}$. Together with the equality on $\bar{\Omega}$, this completes the proof of (3.10).

Remark 3.7. For critical interaction strength $\chi=\chi_{c}$, if $\bar{\rho}$ is a stationary state of Eq. (1.2) with bounded second moment, then it is a global minimiser of $\mathcal{F}_{k}$ by Theorem 3.3. In that case, we can identify the constant $C_{k}[\bar{\rho}]$ in $(2.5)$ with $D_{k}[\bar{\rho}]$ in $(3.9)$, which is the same on all connected components of supp $(\bar{\rho})$.

\subsection{Regularity properties of global minimisers}

Proposition 3.6 allows us to conclude the following useful corollary, adapting some arguments developed in [7].

Corollary 3.8 (Compactly Supported Global Minimisers). If $\chi=\chi_{c}$, then all global minimisers of $\mathcal{F}_{k}$ in $\mathcal{Y}$ are compactly supported. If $0<\chi<\chi_{c}$, then global minimisers of $\mathcal{F}_{k, \text { resc }}$ are compactly supported.

Proof. Let $\rho \in \mathcal{Y}$ be a global minimiser of $\mathcal{F}_{k}$. Then $\rho$ is radially symmetric and non-increasing by Proposition 3.6(i) and has zero energy by (3.6). Using the expression of the constant $D_{k}[\rho]$ given by Proposition 3.6(i), we obtain

$$
D_{k}[\rho]=\frac{m-2}{N(m-1)}\|\rho\|_{m}^{m}<0
$$

Let us assume that $\rho$ is supported on $\mathbb{R}^{N}$. We will arrive at a contradiction by showing that $\rho^{m-1}$ and $W_{k} * \rho$ are in $L^{m /(m-1)}\left(\mathbb{R}^{N}\right)$. Since

$$
D_{k}[\rho]=\frac{m}{N(m-1)} \rho(x)^{m-1}+2 \chi\left(W_{k} * \rho\right)(x)
$$


a.e. in $\mathbb{R}^{N}$ by (3.9), this would mean that the constant $D_{k}[\rho]<0$ is in $L^{m /(m-1)}$ and decays at infinity, which is obviously false.

It remains to show that $W_{k} * \rho$ is in $L^{m /(m-1)}\left(\mathbb{R}^{N}\right)$ since $\rho \in L^{m}\left(\mathbb{R}^{N}\right)$ by assumption. From $\rho \in L^{1}\left(\mathbb{R}^{N}\right) \cap L^{m}\left(\mathbb{R}^{N}\right)$ we have $\rho \in L^{r}\left(\mathbb{R}^{N}\right)$ for all $r \in(1, m]$ by interpolation, and hence $W_{k} * \rho \in L^{s}\left(\mathbb{R}^{N}\right)$ for all $s \in(-N / k, N m /(k(1-m))]$ by [38, Theorem 4.2]. Finally, we conclude that $W_{k} * \rho$ is in $L^{m /(m-1)}\left(\mathbb{R}^{N}\right)$ since $-N / k<m /(m-1)<N m /(k(1-m))$.

In the sub-critical case for the rescaled functional $\mathcal{F}_{k \text {,resc }}$, we argue as above to conclude that for any global minimiser $\rho$ in $\mathcal{Y}_{2}$ we have $\rho^{m-1}$ and $W_{k} * \rho$ in $L^{m /(m-1)}\left(\mathbb{R}^{N}\right)$. If $\rho$ were supported on the whole space, it followed from the Euler-Lagrange condition for the rescaled equation (3.10) that $|x|^{2}+C \in L^{m /(m-1)}\left(\mathbb{R}^{N}\right)$ for some constant $C$. This is obviously false.

The same argument works for stationary states by using the necessary conditions (2.5) and (2.6).

Corollary 3.9 (Compactly Supported Stationary States). If $\chi=\chi_{c}$, then all stationary states of Eq. (1.2) are compactly supported. If $0<\chi<\chi_{c}$, then all stationary states of the rescaled equation (2.3) are compactly supported.

Lemma 3.10. Let $\rho$ be either a global minimiser of $\mathcal{F}_{k}$ over $\mathcal{Y}$ or a global minimiser of $\mathcal{F}_{k, \text { resc }}$ over $\mathcal{Y}_{2}$. If there exists $p \in(-N, 0]$ such that

$$
\rho(r) \lesssim 1+r^{p} \quad \text { for all } r \in(0,1)
$$

then for $r \in(0,1)$,

$$
\rho(r) \lesssim \begin{cases}1+r^{g(p)} & \text { if } p \neq-N-k, \\ 1+|\log (r)|^{\frac{1}{m-1}} & \text { if } p=-N-k,\end{cases}
$$

where

$$
g(p)=\frac{p+N+k}{m-1}
$$

Proof. Since global minimisers are radially symmetric non-increasing, we can bound $\rho(r)$ by $\rho(1)$ for all $r \geq 1$, and hence the bound (3.13) holds true for all $r>0$. Further, we know from Corollary 3.8 that all global minimisers are compactly supported. Let us denote $\operatorname{supp}(\rho)=B(0, R), 0<R<\infty$. We split our analysis in four cases: (1) the regime $1-N<k<0$ with $k \neq 2-N$ and $N \geq 2$, where we can use hypergeometric functions in our estimates, (2) the Newtonian case $k=2-N, N \geq 3$, (3) the one dimensional regime $-1<k<0$ where we need a Cauchy principle value to deal with the singular integral in the mean-field potential gradient, but everything can be computed explicitly, and finally (4) the regime $-N<k \leq 1-N$ and $N \geq 2$, where again singular integrals are needed to deal with the singularities of the hypergeometric functions.

Case $1: 1-N<k<0, k \neq 2-N, N \geq 2$

We would like to make use of the Euler-Lagrange condition (3.9), and hence we need to understand the behaviour of $W_{k} * \rho$. It turns out that it is advantageous to estimate the derivative instead, writing

$$
-\left(W_{k} * \rho\right)(r)=-\left(W_{k} * \rho\right)(1)+\int_{r}^{1} \partial_{r}\left(W_{k} * \rho\right)(s) d s .
$$


The first term on the right-hand side can be estimated explicitly, and we claim that for any $x \in \mathbb{R}^{N}$, we have

$$
-\left(W_{k} * \rho\right)(x)<\infty .
$$

To see this, let us denote $\gamma:=|x|$, and let us fix $\bar{R} \geq R$ such that $0<\gamma<\bar{R}$. If $\gamma / 2 \leq R$, then

$$
\begin{aligned}
-\left(W_{k} * \rho\right)(\gamma) & =\left(-\frac{1}{k}\right) \int_{B(0, R) \backslash B(0, \gamma / 2)}|x-y|^{k} \rho(y) d y+\left(-\frac{1}{k}\right) \int_{B(0, \gamma / 2)}|x-y|^{k} \rho(y) d y \\
& \leq\left(-\frac{1}{k}\right) \rho\left(\frac{\gamma}{2}\right) \int_{B(0, R) \backslash B(0, \gamma / 2)}|x-y|^{k} d y+\left(-\frac{\sigma_{N}}{k}\right) \int_{0}^{\gamma / 2}|\gamma-r|^{k} \rho(r) r^{N-1} d r \\
& \lesssim \gamma^{-N} \int_{B(0, \bar{R}) \backslash B(0, \gamma / 2)}|x-y|^{k} d y+\int_{0}^{\gamma / 2}|\gamma-r|^{k} \rho(r) r^{N-1} d r \\
& \lesssim \gamma^{-N} \int_{B(x, \bar{R}+\gamma)}|x-y|^{k} d y+\left(\frac{\gamma}{2}\right)^{k}\|\rho\|_{1} \\
& =\gamma^{-N} \sigma_{N} \int_{0}^{\bar{R}+\gamma} r^{k+N-1} d r+\left(\frac{\gamma}{2}\right)^{k}<\infty,
\end{aligned}
$$

where we used in the third line the fact that $\rho$ is radially symmetric non-increasing, and so

$$
1 \geq \int_{B(0, \gamma / 2)} \rho(x) d x \geq \rho\left(\frac{\gamma}{2}\right) \int_{B(0, \gamma / 2)} d x=\rho\left(\frac{\gamma}{2}\right)\left(\frac{\sigma_{N}}{N}\right)\left(\frac{\gamma}{2}\right)^{N} .
$$

If $\gamma / 2>R$ on the other hand, we simply have similar to above

$$
-\left(W_{k} * \rho\right)(\gamma) \leq\left(-\frac{1}{k}\right) \int_{B(0, \gamma / 2)}|x-y|^{k} \rho(y) d y \lesssim\left(\frac{\gamma}{2}\right)^{k}<\infty,
$$

which concludes the proof of (3.17).

In order to control the second term in (3.16), we use the formulation (A.3) from the Appendix,

$$
\partial_{r}\left(W_{k} * \rho\right)(r)=r^{k-1} \int_{0}^{\infty} \psi_{k}\left(\frac{\eta}{r}\right) \rho(\eta) \eta^{N-1} d \eta,
$$

where $\psi_{k}$ is given by (A.4) and can be written in terms of Gauss hypergeometric functions, see (A.7).

Sub-Newtonian regime $1-N<k<2-N$

Note that $\psi_{k}(s)<0$ for $s>1$ in the sub-Newtonian regime $1-N<k<2-N$ (see Appendix Lemma A.3 and Fig. 3(a)). Together with the induction assumption (3.13) and using the fact that $\rho$ is compactly supported, we have for any $r \in(0, R)$

$$
\begin{aligned}
\partial_{r}\left(W_{k} * \rho\right)(r) & =r^{k-1} \int_{0}^{r} \psi_{k}\left(\frac{\eta}{r}\right) \rho(\eta) \eta^{N-1} d \eta+r^{k-1} \int_{r}^{R} \psi_{k}\left(\frac{\eta}{r}\right) \rho(\eta) \eta^{N-1} d \eta \\
& =r^{k+N-1} \int_{0}^{1} \psi_{k}(s) \rho(r s) s^{N-1} d s+r^{k+N-1} \int_{1}^{R / r} \psi_{k}(s) \rho(r s) s^{N-1} d s \\
& \leq r^{k+N-1} \int_{0}^{1} \psi_{k}(s) \rho(r s) s^{N-1} d s \\
& \lesssim r^{k+N-1}\left(\int_{0}^{1} \psi_{k}(s) s^{N-1} d s\right)+r^{p+k+N-1}\left(\int_{0}^{1} \psi_{k}(s) s^{p+N-1} d s\right) \\
& =C_{1} r^{k+N-1}+C_{2} r^{p+k+N-1},
\end{aligned}
$$


where we defined

$$
C_{1}:=\int_{0}^{1} \psi_{k}(s) s^{N-1} d s, \quad C_{2}:=\int_{0}^{1} \psi_{k}(s) s^{p+N-1} d s .
$$

In the case when $r \in[R, \infty)$, we use the fact that $\psi_{k}(s)>0$ for $s \in(0,1)$ by Lemma A.3 and so we obtain by the same argument

$$
\begin{aligned}
\partial_{r}\left(W_{k} * \rho\right)(r) & =r^{k-1} \int_{0}^{R} \psi_{k}\left(\frac{\eta}{r}\right) \rho(\eta) \eta^{N-1} d \eta \lesssim r^{k-1} \int_{0}^{R} \psi_{k}\left(\frac{\eta}{r}\right)\left(1+\eta^{p}\right) \eta^{N-1} d \eta \\
& =r^{k+N-1}\left(\int_{0}^{R / r} \psi_{k}(s) s^{N-1} d s\right)+r^{p+k+N-1}\left(\int_{0}^{R / r} \psi_{k}(s) s^{p+N-1} d s\right) \\
& \leq C_{1} r^{k+N-1}+C_{2} r^{p+k+N-1},
\end{aligned}
$$

with constants $C_{1}, C_{2}$ as given above. It is easy to see that $C_{1}$ and $C_{2}$ are indeed finite. From (A.8) it follows that $\psi_{k}(s) s^{N-1}$ and $\psi_{k}(s) s^{p+N-1}$ are integrable at zero since $-N<p$ and $\psi_{k}$ is continuous on $[0,1)$. Similarly, both expressions are integrable at one using (A.10) in Lemma A.4. Hence, we conclude from (3.19) and (3.20) that for any $r \in(0,1)$,

$$
\partial_{r}\left(W_{k} * \rho\right)(r) \lesssim r^{k+N-1}+r^{p+k+N-1} .
$$

Substituting into the right-hand side of (3.16) and using (3.17) yields

$$
-\left(W_{k} * \rho\right)(r) \lesssim 1+\int_{r}^{1}\left(s^{k+N-1}+s^{p+k+N-1}\right) d s
$$

for any $r \in(0,1)$. It follows that for $p \neq-k-N$,

$$
-\left(W_{k} * \rho\right)(r) \lesssim 1+\frac{1-r^{k+N}}{k+N}+\frac{1-r^{p+k+N}}{p+k+N} \lesssim 1+r^{p+k+N} .
$$

If $p=-k-N$, we have instead

$$
-\left(W_{k} * \rho\right)(r) \lesssim 1+\frac{1-r^{k+N}}{k+N}-\log (r) \lesssim 1+|\log (r)| .
$$

If $\rho$ is a global minimiser of $\mathcal{F}_{k}$, then it satisfies the Euler-Lagrange condition (3.9). Hence, we obtain (3.14) with the function $g(p)$ as defined in (3.15). If $\rho$ is a global minimiser of the rescaled functional $\mathcal{F}_{k \text {,resc }}$, then it satisfied condition (3.10) instead, and we arrive at the same result.

\section{Super-Newtonian regime $k>2-N$}

In this regime, $\psi_{k}(s)$ is continuous, positive and strictly decreasing for $s>0$ (see Appendix Lemma A.3 and Fig. $3(\mathrm{~b}))$ and hence integrable on $(0, s)$ for any $s>0$. Under the induction assumption (3.13) and using the fact that $\rho$ is compactly supported and radially symmetric non-increasing, we have for any $r \in(0, R)$

$$
\begin{aligned}
\partial_{r}\left(W_{k} * \rho\right)(r) & =r^{k-1} \int_{0}^{R} \psi_{k}\left(\frac{\eta}{r}\right) \rho(\eta) \eta^{N-1} d \eta=r^{N+k-1} \int_{0}^{R / r} \psi_{k}(s) \rho(r s) s^{N-1} d \eta \\
& \lesssim r^{k+N-1}\left(\int_{0}^{R / r} \psi_{k}(s) s^{N-1} d s\right)+r^{p+k+N-1}\left(\int_{0}^{R / r} \psi_{k}(s) s^{p+N-1} d s\right) \\
& =C_{1}(r) r^{k+N-1}+C_{2}(r) r^{p+k+N-1},
\end{aligned}
$$

where we defined

$$
C_{1}(r):=\int_{0}^{R / r} \psi_{k}(s) s^{N-1} d s, \quad C_{2}(r):=\int_{0}^{R / r} \psi_{k}(s) s^{p+N-1} d s .
$$


Next, let us verify that $C_{1}(\cdot)$ and $C_{2}(\cdot)$ are indeed bounded above. From (A.8) it follows again that $\psi_{k}(s) s^{N-1}$ and $\psi_{k}(s) s^{p+N-1}$ are integrable at zero since $-N<p$. In order to deal with the upper limit, we make use of property (A.9), which implies that there exist constants $L>1$ and $C_{L}>0$ such that for all $s \geq L$, we have

$$
\psi_{k}(s) \leq C_{L} s^{k-2}
$$

It then follows that for $r<R / L$,

$$
\int_{L}^{R / r} \psi_{k}(s) s^{N-1} d s \leq \frac{C_{L}}{N+k-2}\left(\left(\frac{R}{r}\right)^{N+k-2}-L^{N+k-2}\right),
$$

and hence we obtain

$$
C_{1}(r)=\int_{0}^{L} \psi_{k}(s) s^{N-1} d s+\int_{L}^{R / r} \psi_{k}(s) s^{N-1} d s \lesssim 1+r^{-N-k+2} .
$$

Similarly,

$$
C_{2}(r)=\int_{0}^{L} \psi_{k}(s) s^{p+N-1} d s+\int_{L}^{R / r} \psi_{k}(s) s^{p+N-1} d s \lesssim 1+r^{-p-N-k+2}
$$

We conclude

$$
\begin{aligned}
\partial_{r}\left(W_{k} * \rho\right)(r) & \lesssim\left(1+r^{-N-k+2}\right) r^{k+N-1}+\left(1+r^{-p-N-k+2}\right) r^{p+k+N-1} \\
& \lesssim 1+r^{k+N-1}+r^{p+k+N-1} .
\end{aligned}
$$

For $R / L \leq r<R$ on the other hand we can do an even simpler bound:

$$
C_{1}(r)+C_{2}(r) \leq \int_{0}^{L} \psi_{k}(s) s^{N-1} d s+\int_{0}^{L} \psi_{k}(s) s^{p+N-1} d s \lesssim 1,
$$

and so we can conclude for (3.21) directly. In the case when $r \in[R, \infty)$, we obtain by the same argument

$$
\begin{aligned}
\partial_{r}\left(W_{k} * \rho\right)(r) & \lesssim r^{k+N-1}\left(\int_{0}^{R / r} \psi_{k}(s) s^{N-1} d s\right)+r^{p+k+N-1}\left(\int_{0}^{R / r} \psi_{k}(s) s^{p+N-1} d s\right) \\
& \leq C_{1}(R) r^{k+N-1}+C_{2}(R) r^{p+k+N-1},
\end{aligned}
$$

with constants $C_{1}(\cdot), C_{2}(\cdot)$ as given above, and so we conclude that the estimate (3.21) holds true for any $r>0$. Substituting (3.21) into (3.16), we obtain for $r \in(0,1)$

$$
-\left(W_{k} * \rho\right)(r) \lesssim 1+\int_{r}^{1}\left(s^{k+N-1}+s^{p+k+N-1}\right) d s
$$

and so we conclude as in the sub-Newtonian regime.

$$
\text { Case } 2: k=2-N, N \geq 3
$$

Newtonian regime

In the Newtonian case, we can make use of known explicit expressions. We write as above

$$
-\left(W_{k} * \rho\right)(r)=-\left(\frac{r^{2-N}}{(2-N)} * \rho\right)(1)+\int_{r}^{1} \partial_{r}\left(\frac{r^{2-N}}{(2-N)} * \rho\right)(s) d s,
$$

where $-\left(\frac{r^{2-N}}{(2-N)} * \rho\right)(1)$ is bounded using (3.17). To control $\int_{r}^{1} \partial_{r}\left(\frac{r^{2-N}}{(2-N)} * \rho\right)(s) d s$, we use Newton's Shell Theorem implying

$$
\partial_{r}\left(\frac{r^{2-N}}{(2-N)} * \rho\right)(s)=\frac{\sigma_{N} M(s)}{|\partial B(0, s)|}=M(s) s^{1-N},
$$


where we denote by $M(s)=\sigma_{N} \int_{0}^{s} \rho(t) t^{N-1} d t$ the mass of $\rho$ in $B(0, s)$. Note that this is precisely expression (3.18) we obtained in the previous case, choosing $\psi_{k}(s)=1$ for $s<1$ and $\psi_{k}=0$ for $s>1$ with a jump singularity at $s=1$ (see also (A.1) in the Appendix). By our induction assumption (3.13), we have

$$
M(s) \lesssim \sigma_{N} \int_{0}^{s}\left(1+t^{p}\right) t^{N-1} d t=\sigma_{N}\left(\frac{s^{N}}{N}+\frac{s^{N+p}}{N+p}\right), \quad s \in(0,1)
$$

and hence if $p \neq-2$, then

$$
\int_{r}^{1} \partial_{r}\left(\frac{r^{2-N}}{(2-N)} * \rho\right)(s) d s \lesssim \frac{1}{2 N}\left(1-r^{2}\right)+\frac{1}{(N+p)(p+2)}\left(1-r^{p+2}\right) .
$$

If $p=-2$, we obtain instead

$$
\int_{r}^{1} \partial_{r}\left(\frac{r^{2-N}}{(2-N)} * \rho\right)(s) d s \lesssim \frac{1}{2 N}\left(1-r^{2}\right)-\frac{1}{(N+p)} \log (r)
$$

Substituting into the right-hand side of (3.22) yields for all $r \in(0,1)$

$$
-\left(W_{k} * \rho\right)(r) \lesssim \begin{cases}1+r^{p+2} & \text { if } p \neq-2 \\ 1+|\log (r)| & \text { if } p=-2\end{cases}
$$

Thanks again to the Euler-Lagrange condition (3.9) if $\rho$ is a global minimiser of $\mathcal{F}_{k}$, or thanks to condition (3.10) if $\rho$ is a global minimiser of $\mathcal{F}_{k, \text { resc }}$ instead, we arrive in both cases at (3.14).

Case $3:-1<k<0, N=1$

In one dimension, we can calculate everything explicitly. Since the mean-field potential gradient is a singular integral, we have

$$
\begin{aligned}
\partial_{x} S_{k}(x) & =\int_{\mathbb{R}} \frac{x-y}{|x-y|^{2-k}}(\rho(y)-\rho(x)) d y \\
& =\lim _{\delta \rightarrow 0} \int_{|x-y|>\delta} \frac{x-y}{|x-y|^{2-k}} \rho(y) d y=\frac{x}{r} \partial_{r} S_{k}(r)
\end{aligned}
$$

with the radial component for $r \in(0, R)$ given by

$$
\begin{aligned}
\partial_{r} S_{k}(r) & =\int_{0}^{\infty}\left(\frac{r-\eta}{|r-\eta|^{2-k}}+\frac{r+\eta}{|r+\eta|^{2-k}}\right)(\rho(\eta)-\rho(r)) d \eta \\
& =\int_{0}^{\infty} \frac{r+\eta}{|r+\eta|^{2-k}} \rho(\eta) d \eta+\lim _{\delta \rightarrow 0} \int_{|r-\eta|>\delta} \frac{r-\eta}{|r-\eta|^{2-k}} \rho(\eta) d \eta \\
& =r^{k-1} \int_{0}^{\infty} \psi_{1}\left(\frac{\eta}{r}\right) \rho(\eta) d \eta+r^{k-1} \lim _{\delta \rightarrow 0} \int_{|r-\eta|>\delta} \psi_{2}\left(\frac{\eta}{r}\right) \rho(\eta) d \eta \\
& =r^{k-1} \int_{0}^{R} \psi_{1}\left(\frac{\eta}{r}\right) \rho(\eta) d \eta+r^{k-1} \lim _{\delta \rightarrow 0}\left(\int_{0}^{r-\delta}+\int_{r+\delta}^{R}\right) \psi_{2}\left(\frac{\eta}{r}\right) \rho(\eta) d \eta
\end{aligned}
$$

where

$$
\psi_{1}(s):=\frac{1+s}{|1+s|^{2-k}}=(1+s)^{k-1}, \quad \psi_{2}(s):=\frac{1-s}{|1-s|^{2-k}}= \begin{cases}(1-s)^{k-1} & \text { if } 0 \leq s<1 \\ -(s-1)^{k-1} & \text { if } s>1\end{cases}
$$


are well defined on $[0,1) \cup(1, \infty)$. Define $\gamma:=\min \{1, R / 2\}$. Since $\psi_{2}(s)<0$ for $s>1$ and $\rho$ radially symmetric decreasing, we can estimate the last term for any $r \in(0, \gamma)$ and small $\delta>0$ by

$$
\begin{aligned}
r^{k-1} & \left(\int_{0}^{r-\delta}+\int_{r+\delta}^{R}\right) \psi_{2}\left(\frac{\eta}{r}\right) \rho(\eta) d \eta \\
& \leq r^{k-1} \int_{0}^{r-\delta} \psi_{2}\left(\frac{\eta}{r}\right) \rho(\eta) d \eta+r^{k-1} \rho(2 r) \int_{r+\delta}^{2 r} \psi_{2}\left(\frac{\eta}{r}\right) d \eta \\
& =r^{k} \int_{0}^{1-\delta / r} \psi_{2}(s) \rho(r s) d s+r^{k} \rho(2 r) \int_{1+\delta / r}^{2} \psi_{2}(s) d s .
\end{aligned}
$$

Under assumption (3.13), we can bound the above expression by

$$
\begin{aligned}
\partial_{r} S_{k}(r) \lesssim & r^{k} \int_{0}^{R / r} \psi_{1}(s) d s+r^{k+p} \int_{0}^{R / r} \psi_{1}(s) s^{p} d s \\
& +r^{k} \lim _{\delta \rightarrow 0}\left[\int_{0}^{1-\delta / r} \psi_{2}(s) d s+r^{p} \int_{0}^{1-\delta / r} \psi_{2}(s) s^{p} d s+\left(1+r^{p}\right) \int_{1+\delta / r}^{2} \psi_{2}(s) d s\right] \\
= & r^{k} \lim _{\delta \rightarrow 0} C_{1}(r, \delta)+r^{k+p} \lim _{\delta \rightarrow 0} C_{2}(r, \delta),
\end{aligned}
$$

where we defined

$$
\begin{aligned}
& C_{1}(r, \delta):=\int_{0}^{R / r} \psi_{1}(s) d s+\int_{0}^{1-\delta / r} \psi_{2}(s) d s+\int_{1+\delta / r}^{2} \psi_{2}(s) d s \\
& C_{2}(r, \delta):=\int_{0}^{R / r} \psi_{1}(s) s^{p} d s+\int_{0}^{1-\delta / r} \psi_{2}(s) s^{p} d s+\int_{1+\delta / r}^{2} \psi_{2}(s) d s .
\end{aligned}
$$

Next, let us show that the functions $\lim _{\delta \rightarrow 0} C_{1}(r, \delta)$ and $\lim _{\delta \rightarrow 0} C_{2}(r, \delta)$ can be controlled in terms of $r$. The function $\psi_{2}$ has a non-integrable singularity at $s=1$, however, we can seek compensations from below and above the singularity. One can compute directly that

$$
\begin{aligned}
C_{1}(r, \delta) & =\frac{1}{k}\left[\left(\frac{R}{r}+1\right)^{k}-1\right]+\frac{1}{k}\left[1-\left(\frac{\delta}{r}\right)^{k}\right]+\frac{1}{k}\left[\left(\frac{\delta}{r}\right)^{k}-1\right] \\
& =\frac{1}{k}\left(\left(\frac{R}{r}+1\right)^{k}-1\right) \leq-\frac{1}{k} \\
C_{2}(r, \delta) & =\left[\left(\frac{R}{r}+1\right)^{k-1}\left(\frac{R}{r}\right)^{p+1}\right]-\frac{1}{k}\left[\left(\frac{\delta}{r}\right)^{k}\right]+\frac{1}{k}\left[\left(\frac{\delta}{r}\right)^{k}-1\right] \\
& =\left(\frac{R}{r}+1\right)^{k-1}\left(\frac{R}{r}\right)^{p+1}-\frac{1}{k} \leq\left(\frac{R}{r}\right)^{k-1}\left(\frac{R}{r}\right)^{p+1}-\frac{1}{k}=R^{k+p} r^{-k-p}-\frac{1}{k},
\end{aligned}
$$

so that we obtain the estimate

$$
\partial_{r} S_{k}(r) \lesssim 1+r^{k}+r^{k+p}
$$

Finally, we have for all $r \in(0, \gamma)$ :

$$
-\left(W_{k} * \rho\right)(r)=-\left(W_{k} * \rho\right)(\gamma)+\int_{r}^{\gamma} \partial_{r} S_{k}(s) d s \lesssim 1+\int_{r}^{\gamma}\left(s^{k}+s^{p+k}\right) d s,
$$

where we made again use of estimate (3.17). If $p \neq-k-1$, we have

$$
-\left(W_{k} * \rho\right)(r) \lesssim 1+\frac{\gamma^{k+1}-r^{k+1}}{k+1}+\frac{\gamma^{p+k+1}-r^{p+k+1}}{p+k+1} \lesssim 1+r^{p+k+1}
$$


If $p=-k-1$ however, we obtain

$$
-\left(W_{k} * \rho\right)(r) \lesssim 1+\frac{\gamma^{k+1}-r^{k+1}}{k+1}+\log (\gamma)-\log (r) \lesssim 1+|\log (r)| .
$$

Using again the Euler-Lagrange condition (3.9) for a global minimiser of $\mathcal{F}_{k}$ or (3.10) for a global minimiser of $\mathcal{F}_{k, \text { resc }}$ respectively, we obtain (3.14) in the one dimensional case.

Case $4:-N<k \leq 1-N, N \geq 2$

In this case, we can again use hypogeometric functions, but here the mean-field potential gradient is a singular integral due to the singularity properties of hypogeometric functions. It writes as

$$
\nabla S_{k}(x)=\lim _{\delta \rightarrow 0} \int_{|x-y|>\delta} \frac{x-y}{|x-y|^{2-k}} \rho(y) d y=\frac{x}{r} \partial_{r} S_{k}(r)
$$

with the radial component given by

$$
\begin{aligned}
\partial_{r} S_{k}(r) & =r^{k-1} \lim _{\delta \rightarrow 0} \int_{|r-\eta|>\delta} \psi_{k}\left(\frac{\eta}{r}\right) \rho(\eta) \eta^{N-1} d \eta \\
& =r^{k-1} \lim _{\delta \rightarrow 0}\left(\int_{0}^{r-\delta}+\int_{r+\delta}^{R}\right) \psi_{k}\left(\frac{\eta}{r}\right) \rho(\eta) \eta^{N-1} d s
\end{aligned}
$$

where $\psi_{k}$ is given by $(\mathrm{A} .7)$ on $[0,1) \cup(1, \infty)$, and we used the fact that $\rho$ is compactly supported. In this regime, the singularity at $s=1$ is non-integrable and has to be handled with care. Define $\gamma:=\min \{1, R / 2\}$. Since $\psi_{2}(s)<0$ for $s>1$ (see Appendix Lemma A.3) and since $\rho$ is radially symmetric non-increasing, we can estimate the second integral above for any $r \in(0, \gamma)$ and small $\delta>0$ by

$$
\begin{aligned}
r^{k-1} \int_{r+\delta}^{R} \psi_{k}\left(\frac{\eta}{r}\right) \rho(\eta) \eta^{N-1} d \eta & \leq r^{k-1} \rho(2 r) \int_{r+\delta}^{2 r} \psi_{k}\left(\frac{\eta}{r}\right) \eta^{N-1} d \eta \\
& =r^{N+k-1} \rho(2 r) \int_{1+\delta / r}^{2} \psi_{k}(s) s^{N-1} d s
\end{aligned}
$$

Under assumption (3.13), we can then bound the above expression by

$$
\begin{aligned}
\partial_{r}\left(W_{k} * \rho\right)(r) \lesssim & r^{N+k-1} \lim _{\delta \rightarrow 0}\left[\int_{0}^{1-\delta / r} \psi_{k}(s) s^{N-1} d s+r^{p} \int_{0}^{1-\delta / r} \psi_{k}(s) s^{p+N-1} d s\right. \\
& \left.+\left(1+r^{p}\right) \int_{1+\delta / r}^{2} \psi_{k}(s) s^{N-1} d s\right] \\
= & r^{N+k-1} \lim _{\delta \rightarrow 0} C_{1}(r, \delta)+r^{p+N+k-1} \lim _{\delta \rightarrow 0} C_{2}(r, \delta)
\end{aligned}
$$

where

$$
\begin{aligned}
& C_{1}(r, \delta):=\int_{0}^{1-\delta / r} \psi_{k}(s) s^{N-1} d s+\int_{1+\delta / r}^{2} \psi_{k}(s) s^{N-1} d s, \\
& C_{2}(r, \delta):=\int_{0}^{1-\delta / r} \psi_{k}(s) s^{p+N-1} d s+\int_{1+\delta / r}^{2} \psi_{k}(s) s^{N-1} d s .
\end{aligned}
$$

The crucial step is again to show that $\lim _{\delta \rightarrow 0} C_{1}(r, \delta)$ and $\lim _{\delta \rightarrow 0} C_{2}(r, \delta)$ are well-defined and can be controlled in terms of $r$, seeking compensations from above and below the singularity at $s=1$. Recalling that $\psi_{k}(s) s^{N-1}$ and $\psi_{k}(s) s^{p+N-1}$ are integrable at zero by Lemma A.1 and at any finite value above $s=1$ by continuity, we see that the lower bound 0 and upper bound 2 in the integrals only contribute constants, independent of $r$ and $\delta$. The essential step is therefore to check integrability close to the singularity $s=1$. 
From (A.10) and (A.11) in Lemma A.4(2) in the Appendix, we have for any $\alpha \in \mathbb{R}$ and $s$ close to 1 :

$$
\begin{array}{ll}
s<1: & \psi_{k}(s) s^{\alpha}=K_{1}(1-s)^{N+k-2}+O\left((1-s)^{N+k-1}\right), \\
s>1: & \psi_{k}(s) s^{\alpha}=-K_{1}(s-1)^{N+k-2}+O\left((s-1)^{N+k-1}\right),
\end{array}
$$

where the constant $K_{1}$ is given by (A.12)-(A.13). Hence, for $-N<k<1-N$ we obtain

$$
\begin{aligned}
C_{1}(r, \delta) & \lesssim 1-\frac{K_{1}}{N+k-1}\left(\frac{\delta}{r}\right)^{N+k-1}+\frac{K_{1}}{N+k-1}\left(\frac{\delta}{r}\right)^{N+k-1}+O\left(\left(\frac{\delta}{r}\right)^{N+k}\right) \\
& =1+O\left(\left(\frac{\delta}{r}\right)^{N+k}\right)
\end{aligned}
$$

with exactly the same estimate for $C_{2}(r, \delta)$. Taking the limit $\delta \rightarrow 0$, we see that both terms are bounded by a constant. For $k=1-N$, we obtain similarly that both $C_{1}(r, \delta)$ and $C_{2}(r, \delta)$ are bounded by

$$
1-K_{1} \log \left(\frac{\delta}{r}\right)+K_{1} \log \left(\frac{\delta}{r}\right)+O\left(\left(\frac{\delta}{r}\right)\right)=1+O\left(\left(\frac{\delta}{r}\right)\right)
$$

multiplied by some constant. In other words, for any $r \in(0, \gamma)$ and $-N<k \leq 1-N$ we have

$$
\partial_{r}\left(W_{k} * \rho\right)(r) \lesssim r^{N+k-1}+r^{p+N+k-1} .
$$

Now, we are ready to estimate the behaviour of $\rho$ around the origin using again the Euler-Lagrange condition. To estimate the mean-field potential, we use again (3.17) and write

$$
-\left(W_{k} * \rho\right)(r)=-\left(W_{k} * \rho\right)(\gamma)+\int_{r}^{\gamma} \partial_{r}\left(W_{k} * \rho\right)(s) d s \lesssim 1+\int_{r}^{\gamma}\left(s^{k+N-1}+s^{p+k+N-1}\right) d s
$$

for any $r \in(0, \gamma)$. It follows that for $p \neq-k-N$,

$$
-\left(W_{k} * \rho\right)(r) \lesssim 1+\frac{\gamma^{k+N}-r^{k+N}}{k+N}+\frac{\gamma^{p+k+N}-r^{p+k+N}}{p+k+N} \lesssim 1+r^{p+k+N}
$$

If $p=-k-N$, we have instead

$$
-\left(W_{k} * \rho\right)(r) \lesssim 1+\frac{\gamma^{k+N}-r^{k+N}}{k+N}+\log (\gamma)-\log (r) \lesssim 1+|\log (r)| .
$$

This concludes the proof of Lemma 3.10 using again Euler-Lagrange condition (3.9) if $\rho$ is a minimiser of $\mathcal{F}_{k}$, or condition (3.10) if $\rho$ is a minimiser of $\mathcal{F}_{k, \text { resc }}$, to obtain $(3.14)$.

Corollary 3.11 (Boundedness). If $\chi=\chi_{c}$ and $\rho$ is a global minimiser of $\mathcal{F}_{k}$ over $\mathcal{Y}$, then $\rho \in L^{\infty}\left(\mathbb{R}^{N}\right)$. If $0<\chi<\chi_{c}$ and $\rho$ is a global minimiser of $\mathcal{F}_{k, \text { resc }}$ over $\mathcal{Y}_{2}$, then $\rho \in L^{\infty}\left(\mathbb{R}^{N}\right)$.

Proof. Let $\rho$ be a global minimiser of either $\mathcal{F}_{k}$ over $\mathcal{Y}$, or of $\mathcal{F}_{k \text {,resc }}$ over $\mathcal{Y}_{2}$. Since $\rho$ is radially symmetric non-increasing by Proposition 3.6, it is enough to show that $\rho(0)<\infty$. Following the argument in [19], we use induction to show that there exists some $\alpha>0$ such that for all $r \in(0,1)$ we have

$$
\rho(r) \lesssim 1+r^{\alpha} .
$$

Note that $g(p)$ as defined in (3.15) is a linear function of $p$ with positive slope, and let us denote $g^{(n)}(p)=(g \circ g \cdots \circ g)(p)$. Computing explicitly, we have for all $n \in \mathbb{N}$

$$
g^{(n)}(p)=\frac{N+k}{m-2}+\frac{p(m-2)-N-k}{(m-2)(m-1)^{n}}=-N+\frac{p+N}{(m-1)^{n}},
$$

so that

$$
\lim _{n \rightarrow \infty} g^{(n)}(p)=+\infty \text { for any } p>-N .
$$


Since $\rho(r)^{m}|B(0, r)| \leq\|\rho\|_{m}^{m}<\infty$ we obtain the estimate

$$
\rho(r) \leq C\left(N, m,\|\rho\|_{m}\right) r^{-N / m} \text { for all } r>0 .
$$

It follows that $\rho$ satisfies the induction requirement (3.13) with choice $p_{0}:=-N / m$. Since $p_{0}>-N$ there exists $n_{0} \in \mathbb{N}$ such that $g^{\left(n_{0}\right)}\left(p_{0}\right)>0$ and so we can apply Lemma $3.10 n_{0}$ times. This concludes the proof with $\alpha=g^{\left(n_{0}\right)}\left(p_{0}\right)$. We point out that $p_{0}<-N-k$ and so there is a possibility that $g^{(n)}\left(p_{0}\right)=-N-k$ might occur for some $0<n \leq n_{0}$ : if this happens, the logarithmic case occurs and by the second bound in (3.14), we obtain

$$
\rho(r) \lesssim 1+|\log (r)|^{\frac{1}{m-1}} \leq 1+r^{-1}
$$

hence applying the first bound in (3.14) for $p=-1$ yields (3.23) with $\alpha=1 /(m-1)$.

Corollary 3.12 (Regularity). If $\chi=\chi_{c}$, then all global minimisers $\rho \in \mathcal{Y}$ of $\mathcal{F}_{k}$ satisfy $S_{k} \in \mathcal{W}^{1, \infty}\left(\mathbb{R}^{N}\right)$ and $\rho^{m-1} \in \mathcal{W}^{1, \infty}\left(\mathbb{R}^{N}\right)$. If $0<\chi<\chi_{c}$, then all minimisers $\rho \in \mathcal{Y}_{2}$ of $\mathcal{F}_{k, \text { resc }}$ satisfy $S_{k} \in \mathcal{W}^{1, \infty}\left(\mathbb{R}^{N}\right)$ and $\rho^{m-1} \in \mathcal{W}^{1, \infty}\left(\mathbb{R}^{N}\right)$. In the singular range $-N<k \leq 1-N$, we further obtain $\rho \in C^{0, \alpha}\left(\mathbb{R}^{N}\right)$ with $\alpha \in(1-k-N, 1)$ in both original and rescaled variables.

Proof. Let $\rho$ be a global minimiser either of $\mathcal{F}_{k}$ over $\mathcal{Y}$, or of $\mathcal{F}_{k, \text { resc }}$ over $\mathcal{Y}_{2}$. Then $\rho \in L^{\infty}\left(\mathbb{R}^{N}\right)$ by Corollary 3.11. Let us start by considering the singular regime $-N<k \leq 1-N, N \geq 2$ or $-1<k<0$ for $N=1$. Since $\rho \in L^{1}\left(\mathbb{R}^{N}\right) \cap L^{\infty}\left(\mathbb{R}^{N}\right)$, we have $\rho \in L^{p}\left(\mathbb{R}^{N}\right)$ for any $1<p<\infty$.

Using the fact that $\rho=(-\Delta)^{s} S_{k}$ with fractional exponent $s=(N+k) / 2 \in(0,1 / 2)$, we gain $2 s$ derivatives implying $S_{k} \in \mathcal{W}^{2 s, p}\left(\mathbb{R}^{N}\right)$ for $p \geq 2$ if $N \geq 2$ or for $p>-1 / k$ if $N=1$ by using the HLS inequality for Riesz kernels, see [47, Chapter V]. More precisely, by definition of the Bessel potential space $\mathcal{L}^{2 s, p}\left(\mathbb{R}^{N}\right)$, if $u,(-\Delta)^{s} u \in L^{p}\left(\mathbb{R}^{N}\right)$, then $u \in \mathcal{L}^{2 s, p}\left(\mathbb{R}^{N}\right)$. Since $\mathcal{L}^{2 s, p}\left(\mathbb{R}^{N}\right) \subset \mathcal{W}^{2 s, p}\left(\mathbb{R}^{N}\right)$ for any $p \geq 2$ and $s \in(0,1 / 2)[47$, p. 155 , Theorem $5(\mathrm{~A})]$, we have $u \in \mathcal{W}^{2 s, p}\left(\mathbb{R}^{N}\right)$. Next, we use classical Sobolev embedding, $\mathcal{W}^{2 s, p}\left(\mathbb{R}^{N}\right) \subset C^{0, \beta}\left(\mathbb{R}^{N}\right)$ with $\beta=2 s-N / p$ for $p>\frac{N}{2 s}>2$ if $N \geq 2$ or for $p>\max \left\{\frac{1}{k+1},-\frac{1}{k}\right\}$ if $N=1$, which yields $\rho \in C^{0, \beta}\left(\mathbb{R}^{N}\right)$. If $N \geq 2$ and $s=1 / 2$, we use instead that $S_{k} \in \mathcal{L}^{1, p}\left(\mathbb{R}^{N}\right)$ for all $p \geq 2$ implies $S_{k} \in \mathcal{L}^{2 r, p}\left(\mathbb{R}^{N}\right)$ for all $p \geq 2$ and $r \in(0,1 / 2)$ [47, p.135], and then reason as above using any $r \in(0,1 / 2)$ instead of $s=1 / 2$. In the case $1 / 2-N<k \leq 1-N$, we can ensure $\beta>1-k-N$ for large enough $p$, obtaining the required Hölder regularity. For $k \leq 1 / 2-N$ on the other hand, we need to bootstrap a bit further. Let us fix $n \in \mathbb{N}, n \geq 2$ such that

$$
\frac{1}{n+1}-N<k \leq \frac{1}{n}-N
$$

and let us define $\beta_{n}:=\beta+(n-1) 2 s=n 2 s-N / p$. Note that $S_{k} \in L^{\infty}\left(\mathbb{R}^{N}\right)$ by Lemma 2.2 , and $\beta_{n-1}+2 s<1$. This allows us to repeatedly apply [46, Proposition 2.8] stating that $\rho \in C^{0, \gamma}\left(\mathbb{R}^{N}\right)$ implies $S_{k} \in C^{0, \gamma+2 s}\left(\mathbb{R}^{N}\right)$ for any $\gamma \in(0,1]$ such that $\gamma+2 s<1$. It then follows that $\rho^{m-1} \in C^{0, \gamma+2 s}\left(\mathbb{R}^{N}\right)$ using the Euler-Lagrange conditions (3.9) and (3.10) respectively and Corollary 3.8. Since $m \in(1,2)$, we conclude $\rho \in C^{0, \gamma+2 s}\left(\mathbb{R}^{N}\right)$. Iterating this argument $(n-1)$ times starting with $\gamma=\beta$, we obtain $\rho \in C^{0, \beta_{n}}\left(\mathbb{R}^{N}\right)$ and choosing $p$ large enough, we have indeed

$$
\beta_{n}>1-k-N
$$

For any $-N<k<0$, we then have $S_{k} \in \mathcal{W}^{1, \infty}\left(\mathbb{R}^{N}\right)$ by Lemma 2.2. It also immediately follows that $\rho^{m-1} \in \mathcal{W}^{1, \infty}\left(\mathbb{R}^{N}\right)$ using the Euler-Lagrange conditions (3.9) and (3.10) respectively, Corollary 3.8 and Lemma 2.2. Since $m \in(1,2)$, we also conclude $\rho \in \mathcal{W}^{1, \infty}\left(\mathbb{R}^{N}\right)$.

Remark 3.13. For proving sufficient Hölder regularity in the singular regime $-N<k \leq 1-N$, one may choose to bootstrap on the fractional Sobolev space $\mathcal{W}^{2 s, p}\left(\mathbb{R}^{N}\right)$ directly, making use of the Euler-Lagrange 
conditions (3.9) and (3.10) respectively to show that $\rho \in \mathcal{W}^{r, p}\left(\mathbb{R}^{N}\right) \Rightarrow S_{k} \in \mathcal{W}^{r+2 s, p}\left(\mathbb{R}^{N}\right)$ with $r>0$ for $p$ large enough depending only on $N$, see [20]. Here, we need that $\mathcal{W}^{r, p}\left(\mathbb{R}^{N}\right)$ is preserved under taking positive parts of a function for $0<r \leq 1$ and compositions with Lipschitz functions since we take the $1 /(m-1)$ power of $\rho$, see [45, Section 3.1].

Theorem 3.14 (Global Minimisers as Stationary States). If $\chi=\chi_{c}$, then all global minimisers of $\mathcal{F}_{k}$ are stationary states of Eq. (1.2). If $0<\chi<\chi_{c}$, then all global minimisers of $\mathcal{F}_{k, \text { resc }}$ are stationary states of the rescaled equation (2.3).

Proof. For $\chi=\chi_{c}$, let $\rho \in \mathcal{Y}$ be a global minimiser of $\mathcal{F}_{k}$. The regularity properties provided by Corollary 3.12 imply that $\nabla \rho^{m}=\frac{m}{m-1} \rho \nabla \rho^{m-1}$ and that $\rho$ is indeed a distributional solution of (2.1) using (3.9). As a consequence, $\rho$ is a stationary state of Eq. (1.2) according to Definition 2.1. A similar argument holds true in the rescaled case for sub-critical $\chi$.

Remark 3.15. As a matter of fact, the recent result of radial symmetry of stationary states [19] applies to the critical case $\chi=\chi_{c}$ in the range $k \in[2-N, 0)$. Together, Theorem 3.3 and Proposition 3.6 show that all stationary states are radially symmetric for the full range $k \in(-N, 0)$. In other words, the homogeneity of the energy functional $\mathcal{F}_{k}$ allows us to extend the result in [19] to $k \in(-N, 2-N)$ and to find a simple alternative proof in the less singular than Newtonian range.

\section{Fast diffusion case $k>0$}

We investigate in this section the case $k \in(0, N)$ and hence $m \in(0,1)$ where the diffusion is fast in regions where the density of particles is low. The main difficulty is that it seems there is no HLS-type inequality in this range which would provide a lower bound on the free energy, and so a different approach is needed than in the porous medium regime. We concentrate here on the radial setting. Let us define $\mathcal{X}$ to be the set

$$
\mathcal{X}:=\left\{\rho \in L_{+}^{1}\left(\mathbb{R}^{N}\right):\|\rho\|_{1}=1, \int x \rho(x) d x=0\right\} .
$$

The following Lemma will be a key ingredient for studying the behaviour in the fast diffusion case.

Lemma 4.1. For $k \in(0, N)$, any radially symmetric non-increasing $\rho \in \mathcal{X}$ with $|x|^{k} \rho \in L^{1}\left(\mathbb{R}^{N}\right)$ satisfies

$$
I_{k}[\rho] \leq\left(W_{k} * \rho\right)(x) \leq \eta\left(\frac{|x|^{k}}{k}+I_{k}[\rho]\right), \quad \forall x \in \mathbb{R}^{N}
$$

with

$$
I_{k}[\rho]:=\int_{\mathbb{R}^{N}} \frac{|x|^{k}}{k} \rho(x) d x, \quad \eta=\max \left\{1,2^{k-1}\right\}
$$

Proof. The bound from above was proven in (2.2). To prove the lower bound in one dimension, we use the symmetry and monotonicity assumption to obtain

$$
\partial_{x}\left(W_{k} * \rho\right)=\frac{1}{k} \int_{y>0}\left(|x-y|^{k}-|x+y|^{k}\right) \partial_{y} \rho d y \geq 0, \quad \forall x \geq 0
$$

since $|x-y|^{k}-|x+y|^{k} \leq 0$ for $x, y \geq 0$. By symmetry of $W_{k} * \rho$ it follows that $\partial_{x}\left(W_{k} * \rho\right)(x) \leq 0$ for all $x \leq 0$ and hence (4.1) holds true in one dimension for the bound from below. 
For $N \geq 2$, note that since both $W_{k}$ and $\rho$ are radial functions, so is the convolution $W_{k} * \rho$. By slight abuse of notation, we write $\left(W_{k} * \rho\right)(r)$. For $r>0$, we have

$$
\begin{aligned}
\int_{B(0, r)} \Delta_{x}\left(W_{k} * \rho\right) d x & =\int_{\partial B(0, r)} \nabla_{x}\left(W_{k} * \rho\right) \cdot \bar{n} d S \\
& =|\partial B(0, r)| \partial_{r}\left(W_{k} * \rho\right)=r^{N-1} \sigma_{N} \partial_{r}\left(W_{k} * \rho\right) .
\end{aligned}
$$

From $\Delta_{x} W_{k}(x)=(N+k-2)|x|^{k-2}>0$, it then follows that $\partial_{r}\left(W_{k} * \rho\right)(r)>0$ for all $r>0$. This implies the lower bound in higher dimensions.

\subsection{Results in original variables}

Theorem 4.2 (Non-Existence of Stationary States). Let $k \in(0, N)$. For any $\chi>0$, there are no radially symmetric non-increasing stationary states in $\mathcal{X}$ for Eq. (1.2) with kth moment bounded.

Proof. Assume $\bar{\rho} \in \mathcal{X}$ is a radially symmetric non-increasing stationary state for Eq. (1.2) such that $|x|^{k} \bar{\rho} \in L^{1}\left(\mathbb{R}^{N}\right)$. Then $\bar{\rho}$ is continuous by Lemma 2.4. We claim that $\bar{\rho}$ is supported on $\mathbb{R}^{N}$ and satisfies

$$
\bar{\rho}(x)=\left(A W_{k} * \bar{\rho}(x)+C[\bar{\rho}]\right)^{-N / k}, \quad \text { a.e. } x \in \mathbb{R}^{N},
$$

with $A:=2 \chi N k /(N-k)>0$ and some suitably chosen constant $C[\bar{\rho}]$. Indeed, by radiality and monotonicity, supp $(\bar{\rho})=B(0, R)$ for some $R \in(0, \infty]$ and by the same arguments as in Corollary 2.5 leading to (2.7), we obtain

$$
\bar{\rho}(x)^{-k / N}=A W_{k} * \bar{\rho}(x)+C[\bar{\rho}], \quad \text { a.e. } x \in B(0, R) .
$$

Assume $\bar{\rho}$ has compact support, $R<\infty$. It then follows from Lemma 4.1 that the left-hand side is bounded above,

$$
\bar{\rho}(x)^{-k / N} \leq \eta A I_{k}[\bar{\rho}]+\frac{\eta A R^{k}}{k}+C[\bar{\rho}], \quad \text { a.e. } x \in B(0, R) .
$$

By continuity, $\rho(x) \rightarrow 0$ as $|x| \rightarrow R$, but then $\rho(x)^{-k / N}$ diverges, contradicting the bound from above. We must therefore have $R=\infty$, which concludes the proof of (4.2).

Next, taking the limit $x \rightarrow 0$ in (4.2) yields

$$
A I_{k}[\bar{\rho}]+C[\bar{\rho}]>0 .
$$

We then have from Lemma 4.1 for a.e. $x \in \mathbb{R}^{N}$,

$$
0 \leq\left(A \eta\left(\frac{|x|^{k}}{k}+I_{k}[\bar{\rho}]\right)+C[\bar{\rho}]\right)^{-N / k} \leq \bar{\rho}(x) .
$$

However, the lower bound in the estimate above is not integrable on $\mathbb{R}^{N}$, and hence $\bar{\rho} \notin L^{1}\left(\mathbb{R}^{N}\right)$. This contradicts $\bar{\rho} \in \mathcal{X}$.

In the fast diffusion regime, we do not have a suitable HLS-type inequality to show boundedness of the energy functional $\mathcal{F}_{k}$. Although we do not know whether $\mathcal{F}_{k}$ is bounded below or not, we can show that the infimum is not achieved in the radial setting.

Theorem 4.3 (Non-Existence of Global Minimisers). Let $k \in(0, N)$. For any $\chi>0$, there are no radially symmetric non-increasing global minimisers of $\mathcal{F}_{k}$ over $\mathcal{Y}_{k}$. 
Proof. Let $\rho$ be a global minimiser of $\mathcal{F}_{k}$ over $\mathcal{Y}_{k}$. Following the same argument as in Proposition 3.6, we obtain

$$
\begin{array}{ll}
\rho(x)^{-k / N}=A\left(W_{k} * \rho\right)(x)+D_{k}[\rho] & \text { a.e. } \operatorname{in} \operatorname{supp}(\rho), \\
\rho(x)^{-k / N} \geq A\left(W_{k} * \rho\right)(x)+D_{k}[\rho] & \text { a.e. in } \mathbb{R}^{N} .
\end{array}
$$

where

$$
D_{k}[\rho]:=-\frac{2 N k}{(N-k)} \mathcal{F}_{k}[\rho]-\left(\frac{N+k}{N-k}\right) \int_{\mathbb{R}^{N}} \bar{\rho}^{m}(x) d x .
$$

Since $W_{k}$ is continuous and $\rho \in L^{1}\left(\mathbb{R}^{N}\right)$, it follows from (4.3) that $\rho$ is continuous inside its support, being a continuous function of $W_{k}$ convolved with $\rho$. If $\rho$ is radially symmetric non-increasing, then $\operatorname{supp}(\rho)=B(0, R)$ for some $R \in(0, \infty]$. By continuity of $\rho$ at the origin, we can take the limit $|x| \rightarrow 0$ in (4.3) to obtain $A I_{k}[\rho]+D_{k}[\rho]>0$. It then follows from (4.4) and (4.1) that in fact $\rho(x)^{-k / N}>0$ for a.e. $x \in \mathbb{R}^{N}$. Hence, we conclude that $\operatorname{supp}(\rho)=\mathbb{R}^{N}$. The Euler-Lagrange condition (4.3) and estimate (4.1) yield

$$
\rho(x)=\left(A\left(W_{k} * \rho\right)(x)+D_{k}[\rho]\right)^{-N / k} \geq\left(A \eta\left(\frac{|x|^{k}}{k}+I_{k}[\rho]\right)+D_{k}[\rho]\right)^{-N / k}
$$

a.e. on $\mathbb{R}^{N}$. Again, the right-hand side is not integrable for any $k \in(0, N)$ and hence $\rho \notin \mathcal{Y}_{k}$.

\subsection{Results in rescaled variables}

Corollary 4.4 (Necessary Condition for Stationary States). Let $k \in(0, N), \chi>0$ and $\bar{\rho} \in \mathcal{X}$. If $\bar{\rho}$ is a radially symmetric non-increasing stationary state of the rescaled equation (2.3) with bounded kth moment, then $\bar{\rho}$ is continuous, supported on $\mathbb{R}^{N}$ and satisfies

$$
\bar{\rho}(x)=\left(A\left(W_{k} * \bar{\rho}\right)(x)+B|x|^{2}+C[\bar{\rho}]\right)^{-N / k}, \quad \text { a.e. } x \in \mathbb{R}^{N} .
$$

Here, the constant $C[\bar{\rho}]$ is chosen such that $\bar{\rho}$ integrates to one and

$$
A:=2 \chi \frac{N k}{(N-k)}>0, \quad B:=\frac{N k}{2(N-k)}>0 .
$$

Proof. Continuity follows from Lemma 2.4, and we can show supp $(\bar{\rho})=\mathbb{R}^{N}$ and (4.5) by a similar argument as for (4.2).

From the above analysis, if diffusion is too fast, then there are no stationary states to the rescaled equation (2.3):

Theorem 4.5 (Non-Existence of Stationary States). Let $\chi>0, N \geq 3$ and $k \in[2, N)$, then there are no radially symmetric non-increasing stationary states in $\mathcal{X}$ with $k$ th moment bounded to the rescaled equation (2.3).

Proof. Assume $\bar{\rho} \in \mathcal{X}$ is a radially symmetric non-increasing stationary state such that $|x|^{k} \bar{\rho} \in L^{1}\left(\mathbb{R}^{N}\right)$. It follows from (4.5) and (4.1) that

$$
\bar{\rho}(x) \geq\left(A \eta\left(\frac{|x|^{k}}{k}+I_{k}[\bar{\rho}]\right)+B|x|^{2}+C[\bar{\rho}]\right)^{-N / k} .
$$

However, the lower bound is not integrable on $\mathbb{R}^{N}$ for $k \geq 2$, contradicting $\bar{\rho} \in L^{1}\left(\mathbb{R}^{N}\right)$. 
Remark 4.6. Condition (4.5) tells us that radially symmetric non-increasing stationary states have so-called fat tails for large $r=|x|$. More precisely, Lemma 4.1 shows they behave at least like $r^{-N}$ for large $r$ if $k \geq 2$, whereas $\bar{\rho}(r) \sim r^{-2 N / k}$ for large $r>0$ and for $k<2$. This means there is a critical $k_{c}:=2$ and respectively a critical diffusion exponent $m_{c}:=(N-2) / N$ where a change of behaviour occurs.

For $k<k_{c}$, radially symmetric non-increasing stationary states, if they exist, are integrable and mass is preserved. This restriction on $k$ corresponds exactly to the well-known classical fast diffusion regime $m>m_{c}$ in the case $\chi=0$ [53], where mass escapes to the far field but is still preserved. In our case, the behaviour of the tails is dominated by the non-linear diffusion effects even for $\chi>0$ as for the classical fast-diffusion equation when $m>m_{c}$.

If diffusion is too fast, i.e. $k>k_{c}$ and $m<m_{c}$, then no radially symmetric non-increasing stationary states of the rescaled equation (2.3) exist as stated in Theorem 4.5. It is well known that mass escapes to infinity in the case of the classical fast diffusion equation $(\chi=0)$ and integrable $L^{\infty}$-solutions go extinct in finite time (for a detailed explanation of this phenomenon, see [53, Chapter 5.5]). It would be interesting to explore this in our case.

Remark 4.7. If $N \geq 2$ and $k \in\left[k^{*}, 2\right)$ with

$$
k^{*}(N):=-\frac{N}{2}+\sqrt{\frac{N^{2}}{4}+2 N} \in[1,2),
$$

then radially symmetric non-increasing solutions $\bar{\rho} \in \mathcal{X}$ to Eq. (4.5) have unbounded $k$ th moment. Indeed, assuming for a contradiction that $|x|^{k} \bar{\rho} \in L^{1}\left(\mathbb{R}^{N}\right)$. It then follows from (4.5) and (4.1) that

$$
|x|^{k} \bar{\rho}(x) \geq|x|^{k}\left(A \eta\left(\frac{|x|^{k}}{k}+I_{k}[\bar{\rho}]\right)+B|x|^{2}+C[\bar{\rho}]\right)^{-N / k}
$$

a.e. on $\mathbb{R}^{N}$, and the right-hand side is integrable only in the region $k^{2}+N k-2 N<0$. This condition yields (4.7).

Proposition 4.8 (Necessary Condition for Global Minimisers). For $k \in(0, N)$, let $\rho$ be a global minimiser of $\mathcal{F}_{k, \text { resc }}$ in $\mathcal{Y}_{2, k}$. Then for any $\chi>0, \rho$ is continuous inside its support and satisfies

$$
\begin{aligned}
& \rho(x)^{-k / N}=A\left(W_{k} * \rho\right)(x)+B|x|^{2}+D_{k, \operatorname{resc}}[\rho] \quad \text { a.e. in } \operatorname{supp}(\rho), \\
& \rho(x)^{-k / N} \geq A\left(W_{k} * \rho\right)(x)+B|x|^{2}+D_{k, \operatorname{resc}}[\rho] \quad \text { a.e. in } \mathbb{R}^{N} .
\end{aligned}
$$

Here, constants $A, B$ are given by (4.6) and

$$
D_{k, \mathbf{r e s c}}[\rho]:=-4 B \mathcal{F}_{k, \mathbf{r e s c}}[\rho]+B \mathcal{V}[\rho]-\left(\frac{N+k}{N-k}\right) \int_{\mathbb{R}^{N}} \bar{\rho}^{m}(x) d x .
$$

Moreover, radially symmetric non-increasing global minimisers in $\mathcal{Y}_{2 . k}$ are supported on the whole space, and so in that case (4.8) holds true in $\mathbb{R}^{N}$.

Proof. The proof of (4.8) and (4.9) follows analogously to Proposition 3.6. Further, since $W_{k}$ is continuous and $\rho \in L^{1}\left(\mathbb{R}^{N}\right)$, it follows from (4.8) that $\rho$ is continuous inside its support being a continuous function of the convolution between $W_{k}$ and $\rho$. Now, if $\rho$ is radially symmetric non-increasing, we argue as for Theorem 4.3 to conclude that $\operatorname{supp}(\rho)=\mathbb{R}^{N}$.

Remark 4.9. Just like (4.5), condition (4.8) provides the behaviour of the tails for radially symmetric nonincreasing global minimisers of $\mathcal{F}_{k, \text { resc }}$ using the bounds in Lemma 4.1. In particular, they have unbounded 
$k$ th moment for any $\chi>0$ if $k \geq k^{*}$ with $k^{*}$ given by (4.7), and they are not integrable for $k>k_{c}:=2$. Further, their second moment is bounded and $\rho^{m} \in L^{1}\left(\mathbb{R}^{N}\right)$ if and only if $k<2 N /(2+N)$. Note that

$$
\frac{2 N}{2+N}<k^{*}(N)<k_{c}
$$

Hence, radially symmetric non-increasing global minimisers with finite energy $\mathcal{F}_{k, \text { resc }}[\rho]<\infty$ can only exist in the range $0<k<2 N /(2+N)$. For $k \geq \frac{2 N}{2+N}$, one may have to work with relative entropies instead.

Apart from the Euler-Lagrange condition above, we have very little information about global minimisers of $\mathcal{F}_{k, \text { resc }}$ in general, and it is not known in general if solutions to (4.8)-(4.9) exist. Thus, we use a different approach here than in the porous medium regime, showing existence of stationary states to (2.3) directly by a compactness argument. Let us define the set

$$
\overline{\mathcal{X}}:=\left\{\rho \in C\left(\mathbb{R}^{N}\right) \cap \mathcal{X}: \int|x|^{k} \rho(x) d x<\infty, \rho^{*}=\rho, \lim _{r \rightarrow \infty} \rho(r)=0\right\},
$$

where $\rho^{*}$ denotes the symmetric decreasing rearrangement of $\rho$.

Theorem 4.10 (Existence of Stationary States). Let $\chi>0$ and $k \in(0,1] \cap(0, N)$. Then there exists a stationary state $\bar{\rho} \in \overline{\mathcal{X}}$ for the rescaled system (2.3).

Here, decay at infinity of the equilibrium distribution is a property we gain automatically thanks to the properties of the equation, but we choose to include it here a priori.

Proof. Corollary 4.4 suggests that we are looking for a fixed point of the operator $T: \mathcal{X} \rightarrow \mathcal{X}$,

$$
T \rho(x):=\left(A\left(W_{k} * \rho\right)(x)+B|x|^{2}+C\right)^{-N / k} .
$$

For this operator to be well-defined, we need to be able to choose a constant $C=C[\rho]$ such that $\int_{\mathbb{R}} T \rho(x) d x=1$. To show that this is indeed the case, let us define for any $\alpha>0$,

$$
w(\alpha):=\int_{\mathbb{R}^{N}}\left(\alpha+A \frac{|x|^{k}}{k}+B|x|^{2}\right)^{-N / k} d x, \quad W(\alpha):=\int_{\mathbb{R}^{N}}\left(\alpha+B|x|^{2}\right)^{-N / k} d x .
$$

Note that $w$ and $W$ are finite and well-defined since $k<2$. Furthermore, both $w$ and $W$ are continuous, strictly decreasing to zero as $\alpha$ increases, and blow-up at $\alpha=0$. Hence, we can take inverses $\underline{\delta}:=w^{-1}(1)>0$ and $\bar{\delta}:=W^{-1}(1)>0$. Here is where we use the condition $k \leq 1$ as this means $\eta=1$ in Lemma 4.1 (see also Remark 4.11). Fixing some $\rho \in \overline{\mathcal{X}}$ and denoting by $M(\rho, C)$ the mass of $T \rho$, we obtain from Lemma 4.1,

$$
M\left(\rho, \underline{\delta}-A I_{k}[\rho]\right) \geq 1, \quad M\left(\rho, \bar{\delta}-A I_{k}[\rho]\right) \leq 1 .
$$

Since $M(\rho, \cdot)$ is continuous and strictly decreasing on the interval $\left[\underline{\delta}-A I_{k}[\rho], \bar{\delta}-A I_{k}[\rho]\right]$, we conclude that there exists $C[\rho]$ with $\underline{\delta}-A I_{k}[\rho] \leq C[\rho] \leq \bar{\delta}-A I_{k}[\rho]$ and $M(\rho, C[\rho])=1$. From Lemma 4.1, we obtain for all $x \in \mathbb{R}^{N}$,

$$
\left(A I_{k}[\rho]+C[\rho]+A \frac{|x|^{k}}{k}+B|x|^{2}\right)^{-N / k} \leq T \rho(x) \leq\left(A I_{k}[\rho]+C[\rho]+B|x|^{2}\right)^{-N / k},
$$

and integrating over $\mathbb{R}^{N}$,

$$
w\left(A I_{k}[\rho]+C[\rho]\right) \leq 1 \leq W\left(A I_{k}[\rho]+C[\rho]\right)
$$


implying

$$
0<\underline{\delta} \leq A I_{k}[\rho]+C[\rho] \leq \bar{\delta}<\infty
$$

As a consequence, we have a pointwise estimate for $T \rho$,

$$
m(x) \leq T \rho(x) \leq M(x),
$$

where we define

$$
m(x):=\left(\bar{\delta}+A \frac{|x|^{k}}{k}+B|x|^{2}\right)^{-N / k}, \quad M(x):=\left(\underline{\delta}+B|x|^{2}\right)^{-N / k} .
$$

We are now ready to look for a fixed point of $T$. Applying $T$ to $\overline{\mathcal{X}}$, we are able to make use of a variant of the Arzéla-Ascoli Theorem to obtain compactness. The key ingredients are the bounds in Lemma 4.1 and the uniform estimate (4.11) since they allow us to derive the pointwise estimate (4.12), which gives decay at infinity and uniform boundedness of $T \rho$ :

$$
T \rho(x) \leq\left(\underline{\delta}+B|x|^{2}\right)^{-N / k} \leq \min \left\{B^{-N / k}|x|^{-2 N / k}, \underline{\delta}^{-N / k}\right\} .
$$

Further, we claim $T \rho$ is $k$-Hölder continuous on compact balls $K_{R}:=\overline{B(0, R)} \subset \mathbb{R}^{N}, R>0$,

$$
\left|T \rho\left(x_{1}\right)-T \rho\left(x_{2}\right)\right| \leq C_{R, N, k}\left|x_{1}-x_{2}\right|^{k},
$$

with $k$-Hölder semi-norm

$$
[T \rho(\cdot)]_{k}=C_{R, N, k}=\frac{N}{k} \underline{\delta}^{-(1+N / k)}\left(\frac{A}{k}+3 B R^{2-k}\right)>0 .
$$

To see this, let $G(x):=A\left(W_{k} * \rho\right)(x)+B|x|^{2}+C[\rho]$ and $u(G):=G^{-N / k}$ so that we can write

$$
\begin{aligned}
\left|T \rho\left(x_{1}\right)-T \rho\left(x_{2}\right)\right| & =\left|G\left(x_{1}\right)^{-N / k}-G\left(x_{2}\right)^{-N / k}\right| \leq \operatorname{Lip}(u)\left|G\left(x_{1}\right)-G\left(x_{2}\right)\right| \\
& \leq \operatorname{Lip}(u)\left(A\left[W_{k} * \rho\right]_{k}+B\left[|\cdot|^{2}\right]_{k}\right)\left|x_{1}-x_{2}\right|^{k},
\end{aligned}
$$

where $\operatorname{Lip}(\cdot)$ denotes the Lipschitz constant on a suitable domain specified below. Indeed, $G(x)$ satisfies the inequality $0<\underline{\delta} \leq G(x) \leq A \frac{|x|^{k}}{k}+B|x|^{2}+\bar{\delta}$ for all $x \in \mathbb{R}^{N}$ by (4.1) and (4.11). Moreover, $G$ is $k$-Hölder continuous:

$$
\begin{aligned}
\left|\left(W_{k} * \rho\right)\left(x_{1}\right)-\left(W_{k} * \rho\right)\left(x_{2}\right)\right| & =\frac{1}{k} \int_{\mathbb{R}^{N}}|| x_{1}-\left.y\right|^{k}-\left|x_{2}-y\right|^{k} \mid \rho(y) d y \\
& \leq \frac{\left|x_{1}-x_{2}\right|^{k}}{k} 2^{k-1} \leq \frac{\left|x_{1}-x_{2}\right|^{k}}{k}
\end{aligned}
$$

and hence $\left[W_{k} * \rho\right]_{k} \leq 1 / k$ uniformly. Further, the $k$-Hölder semi-norm of $|x|^{2}$ is bounded by $3 R^{2-k}$ on $K_{R}$ : for $x, y \in K_{R}, x \neq y$ and $z:=x-y$, we have for $|z| \leq R$,

$$
\frac{\left.|| x\right|^{2}-|y|^{2} \mid}{|x-y|^{k}} \leq \frac{|z|^{2}+2|z| \min \{|x|,|y|\}}{|z|^{k}} \leq 3 R|z|^{1-k} \leq 3 R^{2-k}
$$

and similarly for $|z| \geq R$,

$$
\frac{\left.|| x\right|^{2}-|y|^{2} \mid}{|x-y|^{k}} \leq \frac{2 R^{2}}{R^{k}}=2 R^{2-k}
$$


and so $\left[|\cdot|^{2}\right]_{k} \leq 3 R^{2-k}$. We are left to estimate the $\operatorname{Lipschitz}$ coefficient $\operatorname{Lip}(u)$ for $G \in[\underline{\delta}, \infty)$. Indeed, we can calculate it explicitly using the mean value theorem,

$$
\left|u\left(G_{1}\right)-u\left(G_{2}\right)\right| \leq\left(\max _{\xi \in[\delta, \infty)}\left|u^{\prime}(\xi)\right|\right)\left|G_{1}-G_{2}\right|,
$$

and so we have

$$
\operatorname{Lip}(u) \leq \max _{\xi \in[\underline{\delta}, \infty)}\left|u^{\prime}(\xi)\right|=\frac{N}{k} \underline{\delta}^{-(1+N / k)} .
$$

This concludes the proof of Hölder continuity of $T \rho$ on $K_{R},(4.15)-(4.16)$. Since $\int_{\mathbb{R}^{N}}|x|^{k} M(x) d x<\infty$ if $k \in(0,1]$, it follows from $(4.12)$ that $T \rho$ has bounded $k$ th moment. Together with the estimate of the tails (4.14), we have indeed $T \overline{\mathcal{X}} \subset \overline{\mathcal{X}}$, and so $T$ is well-defined. We conclude that the operator $T: \overline{\mathcal{X}} \rightarrow \overline{\mathcal{X}}$ is compact by a variant of the Arzéla-Ascoli Theorem using uniform decay at infinity and uniform boundedness (4.14) together with equi-Hölder-continuity (4.15). Continuity of the map $T: \overline{\mathcal{X}} \rightarrow \overline{\mathcal{X}}$ can be analogously checked since the convolution with $W_{k}$ is a continuous map from $\overline{\mathcal{X}}$ to $C\left(\mathbb{R}^{N}\right)$ together with a similar argument as before for the Hölder continuity of $T \rho$. Additionally, we use that $C[\rho]$ is continuous in terms of $\rho$ as $M(\rho, C)$, the mass of $T \rho$, is a continuous function in terms of both $\rho$ and $C$ and strictly decreasing in terms of $C$, and hence $C[\rho]=M^{-1}(\rho, 1)$ is continuous in terms of $\rho$. Here, $M^{-1}(\rho, \cdot)$ denotes the inverse of $M(\rho, \cdot)$.

Finally, by Schauder's fixed point theorem there exists $\bar{\rho} \in \overline{\mathcal{X}}$ such that $T \bar{\rho}=\bar{\rho}$. In other words, $\bar{\rho}$ satisfies relation (4.5) on $\mathbb{R}^{N}$. By continuity and radial monotonicity, we further have $\bar{\rho} \in L^{\infty}\left(\mathbb{R}^{N}\right)$ from which we deduce the required regularity properties using $\operatorname{supp}(\bar{\rho})=\mathbb{R}^{N}$ and Lemma 2.2. We conclude that $\bar{\rho}$ is a stationary state of the rescaled equation according to Definition 2.3.

Remark 4.11. Note that the restriction $k \leq 1$ in the statement of Theorem 4.10 arises from Lemma 4.1 as we need the upper and lower bounds in (4.1) to scale with the same factor $(\eta=1)$. By Corollary 4.4 , this restriction on $k$ also means that we are in the range where stationary states have bounded $k$ th moment since $(0,1] \cap(0, N) \subset\left(0, k^{*}\right)$. To see why this is the case, let us take any $k \in\left(0, k^{*}\right)$ and so $\eta \geq 1$. Applying Lemma 4.1 to $T \rho(x)$ and integrating over $\mathbb{R}^{N}$ then gives

$$
w_{\eta}\left(\eta A I_{k}[\rho]+C[\rho]\right) \leq 1 \leq W\left(A I_{k}[\rho]+C[\rho]\right)
$$

instead of (4.10), with

$$
w_{\eta}(\alpha):=\int_{\mathbb{R}^{N}}\left(\alpha+\eta A \frac{|x|^{k}}{k}+B|x|^{2}\right)^{-N / k} d x .
$$

Taking inverses, we conclude

$$
\underline{\delta}_{\eta} \leq \eta A I_{k}[\rho]+C[\rho], \quad A I_{k}[\rho]+C[\rho] \leq \bar{\delta}
$$

for $\underline{\delta}_{\eta}:=w_{\eta}^{-1}(1)$ and for $\eta \geq 1$. This is where $\eta=1$ becomes necessary in order to conclude for the pointwise estimate (4.12).

If the constant $C[\rho]$ is non-negative however, we can go a bit further and remove the condition $k \leq 1$ whilst still recovering a pointwise estimate on $T \rho$. More precisely, if $C[\rho] \geq 0$, then we obtain from (4.17) for any $k \in\left(0, k^{*}\right)$

$$
0<\frac{\underline{\delta}}{\eta} \leq A I_{k}[\rho]+C[\rho] \leq \bar{\delta}
$$

Instead of (4.12), we get

$$
m_{\eta}(x) \leq T \rho(x) \leq M_{\eta}(x)
$$


with

$$
m_{\eta}(x):=\left(\eta \bar{\delta}+A \frac{|x|^{k}}{k}+B|x|^{2}\right)^{-N / k}, \quad M_{\eta}(x):=\left(\frac{\underline{\delta}_{\eta}}{\eta}+B|x|^{2}\right)^{-N / k} .
$$

However, firstly, the sign of $C[\rho]$ depends on the $k$ th moment $I_{k}[\rho]$, and secondly, knowing a priori that $C[\rho] \geq 0$ implies $C[T \rho] \geq 0$ for all $\rho \in \overline{\mathcal{X}}$ is complicated, see Remark 4.12.

Remark 4.12. Both $C[\rho]<0$ and $C[\rho] \geq 0$ are possible for $\rho \in \overline{\mathcal{X}}$ and $k \in\left(0, k^{*}\right)$, depending on the $k$ th moment of $\rho$. More precisely, $C[\rho]$ is defined as the value in the interval $\left[\underline{\delta}_{\eta}-\eta A I_{k}[\rho], \bar{\delta}-A I_{k}[\rho]\right]$ such that $M(\rho, C[\rho])=1$. Hence, we have

$$
\begin{aligned}
I_{k}[\rho] \leq \underline{\delta}_{\eta} /(\eta A) & \Longrightarrow C[\rho] \geq 0, \\
I_{k}[\rho]>\bar{\delta} / A & \Longrightarrow C[\rho]<0 .
\end{aligned}
$$

Remark 4.13. Having established existence of radially symmetric stationary states to the rescaled equation (2.3), it is a natural question to ask whether these stationary states correspond to minimisers of the rescaled free energy functional $\mathcal{F}_{k \text {,resc }}$. For a stationary state $\bar{\rho}$ to have finite energy, we require in addition $\mathcal{V}[\bar{\rho}]<\infty, \bar{\rho}^{m} \in L^{1}\left(\mathbb{R}^{N}\right)$ and $|x|^{k} \bar{\rho} \in L^{1}\left(\mathbb{R}^{N}\right)$, in which case $\bar{\rho} \in \mathcal{Y}_{2, k}$. As noted in Remark 4.9, this is true if and only if $0<k<\frac{2 N}{2+N}$. This restriction corresponds to $\frac{N}{2+N}<m<1$ and coincides with the regime of the fast diffusion equation $(\chi=0)$ where the Barenblatt profile has second moment bounded and its $m$ th power is integrable [10].

Remark 4.14. In particular, the non-existence result in original variables Theorem 4.3 means that there is no interaction strengths $\chi$ for which the energy functional $\mathcal{F}_{k}$ admits radially symmetric non-increasing global minimisers. In this sense, there is no critical $\chi_{c}$ for $k>0$ as it is the case in the porous medium regime. Existence of global minimisers for the rescaled free energy functional $\mathcal{F}_{k \text {,resc }}$ for all $\chi>0$ would provide a full proof of non-criticality in the fast diffusion range and is still an open problem for arbitrary dimensions $N$. We suspect that $\mathcal{F}_{k \text {,resc }}$ is bounded below. In one dimension, one can establish equivalence between stationary states of the rescaled equation $(2.3)$ and global minimisers of $\mathcal{F}_{k \text {,resc }}$ by completely different methods, proving a type of reversed HLS inequality [13]. The non-existence of a critical parameter $\chi$ is a very interesting phenomenon, which has already been observed in [29] for the one-dimensional limit case $k=1, m=0$.

\subsection{Numerical simulations in one dimension}

To illustrate our analysis of the fast diffusion regime, we present numerical simulations in one dimension. We use a Jordan-Kinderlehrer-Otto (JKO) steepest descent scheme [34,42] which was proposed in [5] for the logarithmic case $k=0$, and generalised to the porous-medium case $k \in(-1,0)$ in [15]. It corresponds to a standard implicit Euler method for the pseudoinverse of the cumulative distribution function, where the solution at each time step of the non-linear system of equations is obtained by an iterative Newton-Raphson procedure. It can easily be extended to rescaled variables and works just in the same way in the fast diffusion regime $k \in(0,1)$.

Our simulations show that solutions in scaled variables for $k \in(0,1)$ converge always to a stationary state suggesting the existence of stationary states as discussed in the previous subsection. Using the numerical scheme, we can do a quality check of the upper and lower bounds derived in (4.12) for stationary states in $\overline{\mathcal{X}}$ :

$$
m(x) \leq \bar{\rho}(x) \leq M(x)
$$




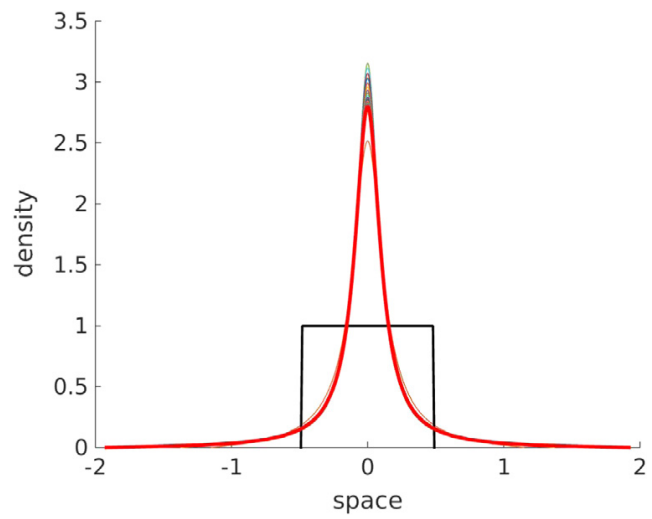

(a) Density.

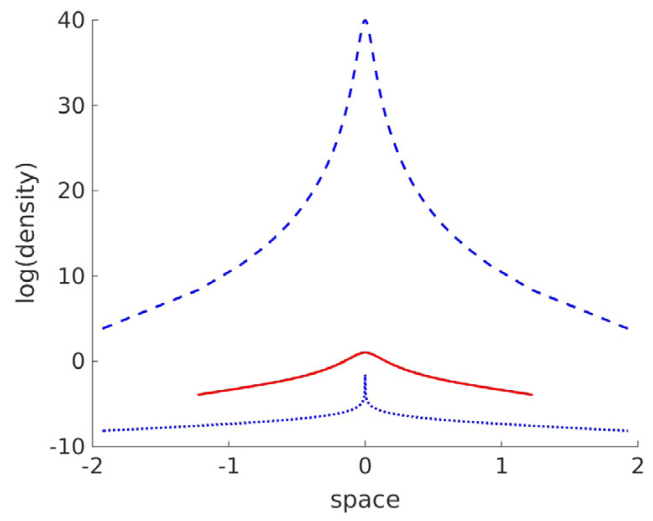

(b) $\log (m(x)) \leq \log (\bar{\rho}(x)) \leq \log (M(x))$.

Fig. 1. Parameter choices: $\chi=1.2, k=0.2$. (A) Density distribution in rescaled variables: As initial data (black) we chose a characteristic supported on the centred ball of radius $1 / 2$, which can be seen to converge to the stationary state $\bar{\rho}$ (red); (B) Logplot of the density including bounds $m(x)$ (dotted blue) and $M(x)$ (dashed blue) as given in (4.12). (For interpretation of the references to colour in this figure legend, the reader is referred to the web version of this article.)

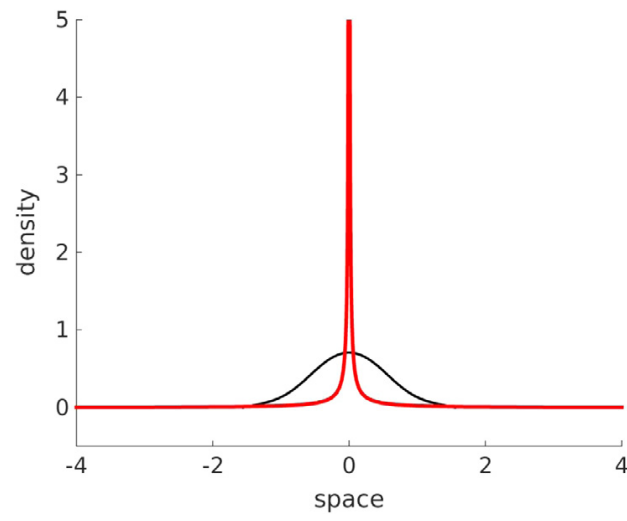

(a) Density.

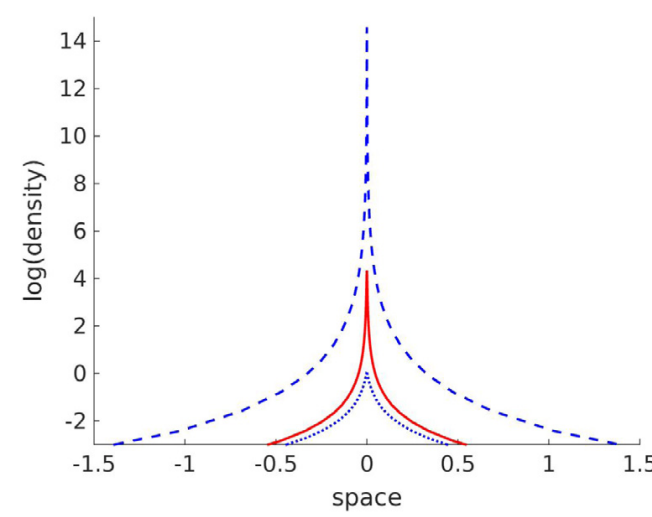

(b) $\log (m(x)) \leq \log (\bar{\rho}(x)) \leq \log (M(x))$.

Fig. 2. Parameter choices: $\chi=0.8, k=0.95$. (A) Density distribution in rescaled variables: As initial data (black) we chose a centred Gaussian distribution, which can be seen to converge to the stationary state $\bar{\rho}$ (red) - here, $\bar{\rho}$ is more peaked as $k$ is closer to 1 and so we only display the lower part of the density plot $\left(\max _{x \in \mathbb{R}} \bar{\rho}(x)=75.7474\right)$; (B) Logplot of the density including bounds $m(x)$ (dotted blue) and $M(x)$ (dashed blue) as given in (4.12). (For interpretation of the references to colour in this figure legend, the reader is referred to the web version of this article.)

with $m(x)$ and $M(x)$ given by (4.13). Figs. 1 and 2 show numerical results at two different points in the $(k, \chi)$-parameter space. For a more detailed description of the numerical scheme and a comprehensive list of numerical results, see [13].

\section{Acknowledgements}

VC received funding for this project from the European Research Council (ERC) under the European Union's Horizon 2020 research and innovation programme (Grant Agreement No 639638). JAC was partially supported by the Royal Society via a Wolfson Research Merit Award and by EPSRC grant number EP/P031587/1. FH acknowledges support from the EPSRC grant number EP/H023348/1 for the Cambridge Centre for Analysis. The authors are very grateful to the Mittag-Leffler Institute for providing a fruitful working environment during the special semester Interactions between Partial Differential Equations 8 Functional Inequalities. 


\section{Appendix. Properties of $\psi_{k}$}

We are here investigating in more detail the properties of the mean-field potential gradient for global minimisers in the porous medium regime. In more than one dimension, it can be expressed in terms of hypergeometric functions. Their properties are well understood and allow us to analyse the regularity properties of global minimisers. Since global minimisers of $\mathcal{F}_{k}$ and $\mathcal{F}_{k \text {,resc }}$ are radially symmetric by Proposition 3.6, the aim is here to find the radial formulation of $\nabla S_{k}$ defined in (1.3). In one dimension, explicit expressions are available, and so we are assuming from now on that $N \geq 2$. There are three different cases: (1) The Newtonian case $k=2-N$ with $N \geq 3$, (2) the range $1-N<k<0, k \neq 2-N$ where $\nabla\left(W_{k} * \rho\right)$ is well defined, and (3) the singular range $-N<k \leq 1-N$ where the force field is given by a Cauchy principle value.

(1) In the Newtonian case $k=2-N$, we have an explicit formula for the radial derivative of the force field using Newton's Shell Theorem,

$$
\partial_{r}\left(W_{2-N} * \rho\right)(r)=M(r) r^{1-N},
$$

where $M(r)=\sigma_{N} \int_{0}^{r} \rho(s) s^{N-1} d s$ is the mass of $\rho$ in a ball of radius $r$. Hence, we can write

$$
\partial_{r}\left(W_{2-N} * \rho\right)(r)=r^{1-N} \int_{0}^{\infty} \psi_{2-N}\left(\frac{\eta}{r}\right) \rho(\eta) \eta^{N-1} \mathrm{~d} \eta
$$

where $\psi_{2-N}$ is defined to have a jump singularity at $s=1$,

$$
\psi_{2-N}(s):= \begin{cases}1 & \text { if } 0 \leq s<1 \\ 0 & \text { if } s>1\end{cases}
$$

(2) In the range $1-N<k<0$ and $k \neq 2-N$, the mean-field potential gradient is given by

$$
\begin{aligned}
\nabla S_{k}(x):=\nabla\left(W_{k} * \rho\right)(x) & =\int_{\mathbb{R}^{N}} \nabla W(x-y) \rho(y) \mathrm{d} y \\
& =\frac{1}{\sigma_{N}} \int_{0}^{\infty} \int_{\partial B(0,|y|)} \nabla W(x-y) \mathrm{d} \sigma(y) \rho(|y|) \mathrm{d}|y| .
\end{aligned}
$$

Denoting $|y|=\eta$, we can write for $x=r e_{1}$,

$$
\begin{aligned}
\frac{1}{\sigma_{N}} \int_{\partial B(0,|y|)} \nabla W(x-y) \mathrm{d} \sigma(y) & =\frac{1}{\sigma_{N}} \int_{\partial B(0,|y|)}(x-y)|x-y|^{k-2} \mathrm{~d} \sigma(y) \\
& =\left(\frac{1}{\sigma_{N}} \int_{\partial B(0, \eta)} e_{1} \cdot\left(r e_{1}-y\right)\left|r e_{1}-y\right|^{k-2} \mathrm{~d} \sigma(y)\right) \frac{x}{r} \\
& =\eta^{N-1}\left(\frac{1}{\sigma_{N}} \int_{\partial B(0,1)}\left(r-\eta e_{1} \cdot z\right)\left|r e_{1}-\eta z\right|^{k-2} \mathrm{~d} \sigma(z)\right) \frac{x}{r} \\
& =\eta^{N-1} r^{k-1} \psi_{k}\left(\frac{\eta}{r}\right) \frac{x}{r},
\end{aligned}
$$

where

$$
\psi_{k}(s)=\frac{1}{\sigma_{N}} \int_{\partial B(0,1)}\left(1-s e_{1} . z\right)\left|e_{1}-s z\right|^{k-2} \mathrm{~d} \sigma(z), \quad s \in[0,1) \cup(1, \infty) .
$$


By radial symmetry,

$$
\nabla\left(W_{k} * \rho\right)(x)=r^{k-1}\left(\int_{0}^{\infty} \psi_{k}\left(\frac{\eta}{r}\right) \rho(\eta) \eta^{N-1} \mathrm{~d} \eta\right) \frac{x}{r}=\partial_{r}\left(W_{k} * \rho\right)(r) \frac{x}{r}
$$

with

$$
\partial_{r}\left(W_{k} * \rho\right)(r)=r^{k-1} \int_{0}^{\infty} \psi_{k}\left(\frac{\eta}{r}\right) \rho(\eta) \eta^{N-1} \mathrm{~d} \eta
$$

(3) In the regime $-N<k \leq 1-N$ however, the derivative of the convolution with the interaction kernel is a singular integral, and in this case the force field is defined as

$$
\begin{aligned}
\nabla S_{k} & :=\int_{\mathbb{R}} \frac{x-y}{|x-y|^{2-k}}(\rho(y)-\rho(x)) d y \\
& =\lim _{\delta \rightarrow 0} \int_{|x-y|>\delta} \frac{x-y}{|x-y|^{2-k}} \rho(y) d y=\frac{x}{r} \partial_{r} S_{k}(r)
\end{aligned}
$$

with the radial component given by

$$
\begin{aligned}
\partial_{r} S_{k}(r) & =r^{k-1} \int_{0}^{\infty} \psi_{k}\left(\frac{\eta}{r}\right)(\rho(\eta)-\rho(r)) \eta^{N-1} d \eta \\
& =r^{k-1} \lim _{\delta \rightarrow 0} \int_{|r-\eta|>\delta} \psi_{k}\left(\frac{\eta}{r}\right) \rho(\eta) \eta^{N-1} d \eta
\end{aligned}
$$

and $\psi_{k}$ is given by $(\mathrm{A} .2)$ on $[0,1) \cup(1, \infty)$.

For any $-N<k<0$ with $k \neq 2-N$, we can rewrite (A.2) as

$$
\psi_{k}(s)=\frac{\sigma_{N-1}}{\sigma_{N}} \int_{0}^{\pi}(1-s \cos (\theta)) \sin ^{N-2}(\theta) A(s, \theta)^{k-2} d \theta, \quad s \in[0,1) \cup(1, \infty)
$$

with

$$
A(s, \theta)=\left(1+s^{2}-2 s \cos (\theta)\right)^{1 / 2} .
$$

It is useful to express $\psi_{k}$ in terms of Gauss Hypergeometric Functions. The hypergeometric function $F(a, b ; c ; z)$ is defined as the power series

$$
F(a, b ; c ; z)=\sum_{n=0}^{\infty} \frac{(a)_{n}(b)_{n}}{(c)_{n}} \frac{z^{n}}{n !}
$$

for $|z|<1$ and $a, b \in \mathbb{C}, c \in \mathbb{C} \backslash\left\{\mathbb{Z}^{-} \cup\{0\}\right\}$, see [2], where $(q)_{n}$ is the Pochhammer symbol defined for any $q>0, n \in \mathbb{N}$ by

$$
(q)_{0}=1, \quad(q)_{n}=\frac{(n+q-1) !}{(q-1) !} .
$$

We will here make use of its well known integral representation [2]

$$
F(a, b ; c ; z)=\frac{\Gamma(c)}{\Gamma(b) \Gamma(c-b)} \int_{0}^{1} t^{b-1}(1-t)^{c-b-1}(1-t z)^{-a} d t
$$

for $c>b>0, a>0$ and $|z|<1$. Moreover, if $c-a-b>0$, then $F$ is well defined at $z=1$ and satisfies

$$
F(a, b ; c ; 1)=\frac{\Gamma(c) \Gamma(c-a-b)}{\Gamma(c-a) \Gamma(c-b)} .
$$


Otherwise, we have the limiting case discussed in [2]:

$$
\lim _{z \rightarrow 1^{-}} \frac{F(a, b ; c ; z)}{(1-z)^{c-a-b}}=\frac{\Gamma(c) \Gamma(a+b-c)}{\Gamma(a) \Gamma(b)}, \quad \text { if } c-a-b<0 .
$$

Let us define

$$
H(a, b ; c ; z):=\frac{\Gamma(b) \Gamma(c-b)}{\Gamma(c)} F(a, b ; c ; z) .
$$

To express $\psi_{k}$ as a combination of hypergeometric functions, we write

$$
\begin{aligned}
\psi_{k}(s)= & \frac{\sigma_{N-1}}{\sigma_{N}} \int_{0}^{\pi}(1-s \cos (\theta))\left(1+s^{2}-2 s \cos (\theta)\right)^{\frac{k-2}{2}} \sin ^{N-2}(\theta) d \theta \\
= & \frac{\sigma_{N-1}}{\sigma_{N}}(1+s)^{k-2} \int_{0}^{\pi}(1-s \cos (\theta))\left(1-\frac{4 s}{(1+s)^{2}} \cos ^{2}\left(\frac{\theta}{2}\right)\right)^{\frac{k-2}{2}} \sin ^{N-2}(\theta) d \theta \\
= & \frac{\sigma_{N-1}}{\sigma_{N}}(1+s)^{k-2} \int_{0}^{\pi}\left(1-\frac{4 s}{(1+s)^{2}} \cos ^{2}\left(\frac{\theta}{2}\right)\right)^{\frac{k-2}{2}} \sin ^{N-2}(\theta) d \theta \\
& -\frac{\sigma_{N-1}}{\sigma_{N}}(1+s)^{k-2} s \int_{0}^{\pi} \cos (\theta)\left(1-\frac{4 s}{(1+s)^{2}} \cos ^{2}\left(\frac{\theta}{2}\right)\right)^{\frac{k-2}{2}} \sin ^{N-2}(\theta) d \theta \\
= & : f_{1}(s)-f_{2}(s) .
\end{aligned}
$$

Now, we use the change of variable $t=\cos ^{2}(\theta / 2)$ to get

$$
\begin{aligned}
f_{1}(s) & =\frac{\sigma_{N-1}}{\sigma_{N}}(1+s)^{k-2} \int_{0}^{\pi}\left(1-\frac{4 s}{(1+s)^{2}} \cos ^{2}\left(\frac{\theta}{2}\right)\right)^{\frac{k-2}{2}} \sin ^{N-2}(\theta) d \theta \\
& =\frac{\sigma_{N-1}}{\sigma_{N}}(1+s)^{k-2} 2^{N-2} \int_{0}^{1}\left(1-\frac{4 s}{(1+s)^{2}} t\right)^{\frac{k-2}{2}} t^{\frac{N-3}{2}}(1-t)^{\frac{N-3}{2}} d t \\
& =\frac{\sigma_{N-1}}{\sigma_{N}}(1+s)^{k-2} 2^{N-2} H\left(a, b_{1} ; c_{1} ; z\right)
\end{aligned}
$$

with

$$
a:=1-\frac{k}{2}, \quad b_{1}:=\frac{N-1}{2}, \quad c_{1}:=N-1, \quad z:=\frac{4 s}{(1+s)^{2}} .
$$

Let us define $h_{1}(s):=f_{1}(s)$, and

$$
h_{2}(s):=\frac{\sigma_{N-1}}{\sigma_{N}}(1+s)^{k-2} 2^{N-2} H\left(a, b_{2} ; c_{2} ; z\right)
$$

with

$$
a:=1-\frac{k}{2}, \quad b_{2}:=\frac{N-1}{2}, \quad c_{2}:=N-1, \quad z:=\frac{4 s}{(1+s)^{2}} .
$$

Then

$$
\begin{aligned}
f_{2}(s) & =\frac{\sigma_{N-1}}{\sigma_{N}}(1+s)^{k-2} s \int_{0}^{\pi} \cos (\theta)\left(1-\frac{4 s}{(1+s)^{2}} \cos ^{2}\left(\frac{\theta}{2}\right)\right)^{\frac{k-2}{2}} \sin ^{N-2}(\theta) d \theta \\
& =-\operatorname{sh}_{1}(s)+2 \operatorname{sh}_{2}(s)
\end{aligned}
$$

by the same change of variable. We conclude

$$
\psi_{k}(s)=(1+s) h_{1}(s)-2 s h_{2}(s), \quad s \in[0,1) \cap(1, \infty) .
$$

Let us now study the behaviour of $\psi_{k}$ in more detail for $k \neq 2-N$. For any fixed $s \in[0,1) \cap(1, \infty)$,

$$
\left|\psi_{k}(s)\right| \leq \frac{1}{\sigma_{N}} \int_{\partial B(0,1)}\left|e_{1}-s x\right|^{k-1} d \sigma(x)<\infty
$$




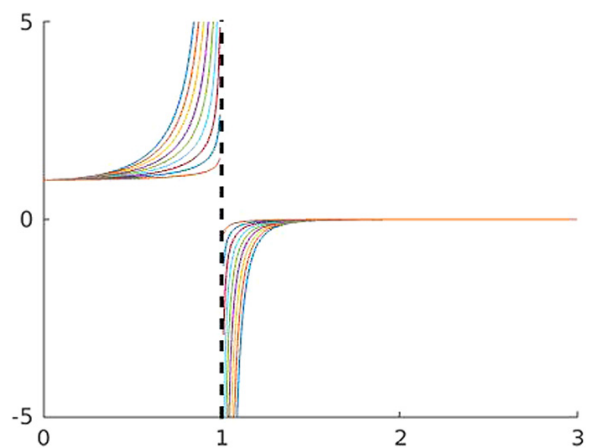

(a) $-N<k<2-N$.

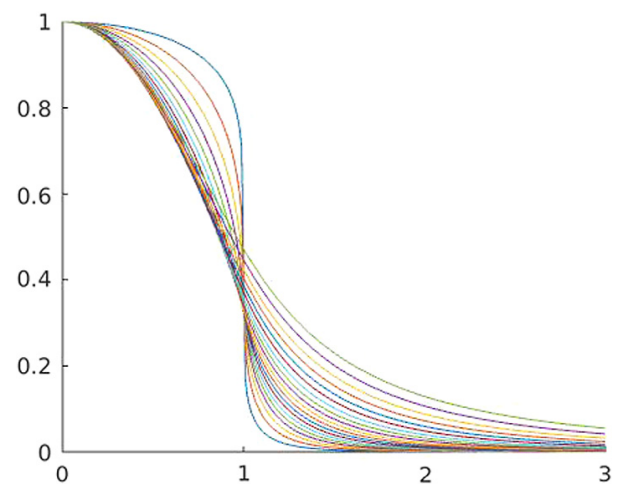

(b) $2-N<k<0$.

Fig. 3. $\psi_{k}$ for different values of $k$ with $N=6$, increasing $k$ by 0.2 for each plot.

and by the dominated convergence theorem, it is easy to see that $\psi_{k}$ is continuous on $s \in[0,1) \cap(1, \infty)$ for any $-N<k<2-N$ and $2-N<k<0$. A singularity occurs at $s=1$ if $k<2-N$, however this singularity is integrable in the range $1-N<k<2-N$.

In order to handle the expression of the mean-field potential gradient, it is important to understand the behaviour of $\psi_{k}$ at the limits of the integral 0 and $\infty$ as well as at the singularity $s=1$.

Lemma A.1 (Behaviour at 0). For $\alpha>-1,-N<k<0$ and small $s>0$,

$$
\psi_{k}(s) s^{\alpha}=s^{\alpha}+O\left(s^{\alpha+1}\right) .
$$

Proof. Following the same argument as in [32, Lemma 4.4], we obtain $\psi_{k}(0)=1$ for any $-N<k<0$, and so (A.8) follows.

Similarly, extending the argument in [32, Lemma 4.4] to $-N<k<0$, we have

Lemma A.2 (Behaviour at $\infty)$. For $-N<k<0$,

$$
\lim _{s \rightarrow \infty} s^{2-k} \psi_{k}(s)=\frac{N+k-2}{N} .
$$

Further, it is obvious from (A.4) that $\psi_{k}(s)>0$ for $s \in(0,1)$. From [32],

$$
\psi_{k}^{\prime}(s)=\left(\frac{\sigma_{N-1}}{\sigma_{N}}\right) \frac{(k-2)(N+k-2)}{(N-1)} s \int_{0}^{\pi} \sin ^{N}(\theta) A(s, \theta)^{k-4} d \theta, \quad s \in[0,1) \cap(1, \infty)
$$

and hence $\psi_{k}$ is strictly decreasing for $k>2-N$ and strictly increasing for $k<2-N$. It then follows from (A.9) that in the super-Newtonian regime $k>2-N, \psi_{k}$ converges to zero as $s \rightarrow \infty$, is finite and continuous at $s=1$, and strictly positive on $[0, \infty$ ) (Fig. 3(b)). In the sub-Newtonian regime $-N<k<2-N$ on the other hand, the monotonicity of $\psi_{k}$ and the fact that $\psi_{k}$ converges to 0 as $s \rightarrow \infty$ imply that

$$
\lim _{s \rightarrow 1^{-}} \psi_{k}(s)=+\infty, \quad \lim _{s \rightarrow 1^{+}} \psi_{k}(s)=-\infty,
$$

and so we conclude that $\psi_{k}<0$ on $(1, \infty)$ if $-N<k<2-N$ (Fig. 3(a)). We summarise these observations in the following lemma:

Lemma A.3 (Overall Behaviour). Let $\psi_{k}$ be as defined in (A.2). 
(i) If $2-N<k<0$, then $\psi_{k}$ is continuous, positive and strictly decreasing on $[0, \infty)$.

(ii) If $-N<k<2-N$, then $\psi_{k}$ is continuous, positive and strictly increasing on $[0,1)$, and it is continuous, negative and strictly increasing on $(1, \infty)$. Further, it has a singularity at $s=1$ which is integrable for $1-N<k<2-N$.

Using the hypergeometric function representation of $\psi_{k}$, we can characterise its behaviour near the singularity.

Lemma A.4 (Behaviour at 1). For $\alpha \in \mathbb{R}$ and $\varepsilon>0$ small, we have

(1) in the super-Newtonian regime $2-N<k<0$ and for $s=1 \pm \varepsilon$ :

$$
\psi_{k}(s) s^{\alpha}=\psi_{k}(1)+O(\varepsilon)
$$

(2) in the sub-Newtonian regime $-N<k<2-N$ and

(i) for $s=1-\varepsilon$ :

$$
\psi_{k}(s) s^{\alpha}=K_{1} \varepsilon^{N+k-2}+K_{2} \varepsilon^{N+k-1}+O\left(\varepsilon^{N+k}\right)
$$

(ii) for $s=1+\varepsilon$ :

$$
\psi_{k}(s) s^{\alpha}=-K_{1} \varepsilon^{N+k-2}+K_{3} \varepsilon^{N+k-1}+O\left(\varepsilon^{N+k}\right),
$$

where

$$
\begin{aligned}
& K_{1}=\left(\frac{\sigma_{N-1}}{\sigma_{N}}\right) \frac{\gamma}{2}>0, \quad K_{2}[\alpha]=-\left(\frac{\sigma_{N-1}}{\sigma_{N}}\right)\left(\frac{B_{1}+\gamma(1-N+2 \alpha)}{4}\right), \\
& K_{3}[\alpha]=-\left(\frac{\sigma_{N-1}}{\sigma_{N}}\right)\left(\frac{B_{1}+\gamma(2 k+N-5+2 \alpha)}{4}\right)
\end{aligned}
$$

and

$$
\gamma=\frac{\Gamma\left(c_{2}-b_{2}\right) \Gamma\left(a+b_{2}-c_{2}\right)}{\Gamma(a)}>0 .
$$

Proof. (1) follows directly from the fact that $\psi_{k}$ is continuous at $s=1$ [32, Lemma 4.4]. In order to prove (2), we make use of expression (A.7) for $\psi_{k}$ in terms of hypergeometric functions and known expansions around the point of singularity. Denoting $\delta:=\varepsilon /|2-\varepsilon|>0$, we have for any $\beta>0$,

$$
\delta^{\beta}=\left(\frac{\varepsilon}{2}\right)^{\beta}+\beta\left(\frac{\varepsilon}{2}\right)^{\beta+1}+O\left(\varepsilon^{\beta+2}\right) .
$$

From (A.7) we can write

$$
\psi_{k}(s)=(1+s) h_{1}(s)-2 s h_{2}(s)=(1+s)\left(h_{1}(s)-h_{2}(s)\right)+(1-s) h_{2}(s),
$$

and hence, denoting $z=1-\delta^{2}$, we obtain for $s=1-\varepsilon$ :

$$
\begin{aligned}
2^{2-N} \frac{\sigma_{N}}{\sigma_{N-1}} \psi_{k}(1-\varepsilon)= & (2-\varepsilon)^{k-1}\left(H\left(a, b_{1} ; c_{1} ; z\right)-H\left(a, b_{2} ; c_{2} ; z\right)\right)+\varepsilon(2-\varepsilon)^{k-2} H\left(a, b_{2} ; c_{2} ; z\right) \\
= & (2-\varepsilon)^{k-1} \delta^{N+k-3}\left(\frac{H\left(a, b_{1} ; c_{1} ; z\right)}{(1-z)^{c_{1}-a-b_{1}}}-\frac{H\left(a, b_{2} ; c_{2} ; z\right)}{(1-z)^{c_{2}-a-b_{2}}}\right) \\
& +\varepsilon(2-\varepsilon)^{k-2} \delta^{N+k-3}\left(\frac{H\left(a, b_{2} ; c_{2} ; z\right)}{(1-z)^{c_{2}-a-b_{2}}}\right)
\end{aligned}
$$


Similarly, above the singularity point at $s=1+\varepsilon$, we obtain:

$$
\begin{aligned}
2^{2-N} \frac{\sigma_{N}}{\sigma_{N-1}} \psi_{k}(1+\varepsilon)= & (2+\varepsilon)^{k-1} \delta^{N+k-3}\left(\frac{H\left(a, b_{1} ; c_{1} ; z\right)}{(1-z)^{c_{1}-a-b_{1}}}-\frac{H\left(a, b_{2} ; c_{2} ; z\right)}{(1-z)^{c_{2}-a-b_{2}}}\right) \\
& -\varepsilon(2+\varepsilon)^{k-2} \delta^{N+k-3}\left(\frac{H\left(a, b_{2} ; c_{2} ; z\right)}{(1-z)^{c_{2}-a-b_{2}}}\right) .
\end{aligned}
$$

Using the power series expression (A.5) for hypergeometric functions, we can write

$$
\begin{aligned}
& \left(\frac{H\left(a, b_{1} ; c_{1} ; z\right)}{(1-z)^{c_{1}-a-b_{1}}}-\frac{H\left(a, b_{2} ; c_{2} ; z\right)}{(1-z)^{c_{2}-a-b_{2}}}\right)=\sum_{n=0}^{\infty} A_{n} \frac{z^{n}}{n !}=\sum_{m=0}^{\infty} \frac{(-1)^{m} B_{m}}{m !} \delta^{2 m}, \\
& B_{m}:=\sum_{n=m}^{\infty} \frac{A_{n}}{(n-m) !}, \\
& A_{n}:=\left(\frac{\left(c_{1}-a\right)_{n}\left(c_{1}-b_{1}\right)_{n}}{\left(c_{1}\right)_{n}}-b_{1} \frac{\left(c_{2}-a\right)_{n}\left(c_{2}-b_{2}\right)_{n}}{\left(c_{2}\right)_{n}}\right) \frac{\Gamma\left(b_{1}\right) \Gamma\left(c_{1}-b_{1}\right)}{\Gamma(a)} .
\end{aligned}
$$

In the singularity regime $-N<k<2-N$, we have

$$
c_{1}-a-b_{1}=c_{2}-a-b_{2}=\frac{N+k-3}{2}<0,
$$

and so we can make use of (A.6) to show that the leading order term vanishes:

$$
\begin{aligned}
B_{0} & =\lim _{\delta \rightarrow 0} \sum_{m=0}^{\infty} \frac{(-1)^{m} B_{m}}{m !} \delta^{2 m}=\lim _{z \rightarrow 1^{-}}\left(\frac{H\left(a, b_{1} ; c_{1} ; z\right)}{(1-z)^{c_{1}-a-b_{1}}}-\frac{H\left(a, b_{2} ; c_{2} ; z\right)}{(1-z)^{c_{2}-a-b_{2}}}\right) \\
& =\frac{\Gamma\left(c_{1}-b_{1}\right) \Gamma\left(a+b_{1}-c_{1}\right)}{\Gamma(a)}-\frac{\Gamma\left(c_{2}-b_{2}\right) \Gamma\left(a+b_{2}-c_{2}\right)}{\Gamma(a)}=0 .
\end{aligned}
$$

Hence

$$
\begin{aligned}
& \frac{H\left(a, b_{1} ; c_{1} ; z\right)}{(1-z)^{c_{1}-a-b_{1}}}-\frac{H\left(a, b_{2} ; c_{2} ; z\right)}{(1-z)^{c_{2}-a-b_{2}}}=-B_{1} \delta^{2}+O\left(\delta^{4}\right), \\
& \frac{H\left(a, b_{2} ; c_{2} ; z\right)}{(1-z)^{c_{2}-a-b_{2}}}=\frac{\Gamma\left(c_{2}-b_{2}\right) \Gamma\left(a+b_{2}-c_{2}\right)}{\Gamma(a)}+O\left(\delta^{2}\right):=\gamma+O\left(\delta^{2}\right) .
\end{aligned}
$$

Substituting these estimates and making use of (A.14), (A.15) becomes

$$
\begin{aligned}
2^{2-N} \frac{\sigma_{N}}{\sigma_{N-1}} \psi_{k}(1-\varepsilon)= & \varepsilon(2-\varepsilon)^{k-2}\left(\gamma \delta^{N+k-3}+O\left(\delta^{N+k-1}\right)\right),+(2-\varepsilon)^{k-1}\left(-B_{1} \delta^{N+k-1}+O\left(\delta^{N+k+1}\right)\right) \\
= & \varepsilon\left[2^{k-2}-\varepsilon(k-2) 2^{k-3}+O\left(\varepsilon^{2}\right)\right] \\
& \times\left[\gamma\left(\frac{\varepsilon}{2}\right)^{N+k-3}+\gamma(N+k-3)\left(\frac{\varepsilon}{2}\right)^{N+k-2}+O\left(\varepsilon^{N+k-1}\right)\right] \\
& +\left[2^{k-1}+O(\varepsilon)\right]\left[-B_{1}\left(\frac{\varepsilon}{2}\right)^{N+k-1}+O\left(\varepsilon^{N+k}\right)\right] \\
= & \gamma 2^{-N+1} \varepsilon^{N+k-2}+\gamma 2^{-N}[(N+k-3)+(2-k)] \varepsilon^{N+k-1} \\
& -B_{1} 2^{-N} \varepsilon^{N+k-1}+O\left(\varepsilon^{N+k}\right) \\
= & \gamma 2^{-N+1} \varepsilon^{N+k-2}+2^{-N}\left[\gamma(N-1)-B_{1}\right] \varepsilon^{N+k-1}+O\left(\varepsilon^{N+k}\right) .
\end{aligned}
$$


Similarly, (A.16) has expansion

$$
\begin{aligned}
2^{2-N} \frac{\sigma_{N}}{\sigma_{N-1}} \psi_{k}(1+\varepsilon)= & -\varepsilon(2+\varepsilon)^{k-2}\left(\gamma \delta^{N+k-3}+O\left(\delta^{N+k-1}\right)\right),+(2+\varepsilon)^{k-1}\left(-B_{1} \delta^{N+k-1}+O\left(\delta^{N+k+1}\right)\right) \\
= & -\varepsilon\left[2^{k-2}+\varepsilon(k-2) 2^{k-3}+O\left(\varepsilon^{2}\right)\right] \\
& \times\left[\gamma\left(\frac{\varepsilon}{2}\right)^{N+k-3}+\gamma(N+k-3)\left(\frac{\varepsilon}{2}\right)^{N+k-2}+O\left(\varepsilon^{N+k-1}\right)\right] \\
& +\left[2^{k-1}+O(\varepsilon)\right]\left[-B_{1}\left(\frac{\varepsilon}{2}\right)^{N+k-1}+O\left(\varepsilon^{N+k}\right)\right] \\
= & -\gamma 2^{-N+1} \varepsilon^{N+k-2}+\gamma 2^{-N}[-(N+k-3)+(2-k)] \varepsilon^{N+k-1} \\
& -B_{1} 2^{-N} \varepsilon^{N+k-1}+O\left(\varepsilon^{N+k}\right) \\
= & -\gamma 2^{-N+1} \varepsilon^{N+k-2}+2^{-N}\left[\gamma(5-N-2 k)-B_{1}\right] \varepsilon^{N+k-1}+O\left(\varepsilon^{N+k}\right) .
\end{aligned}
$$

We conclude

$$
\begin{array}{r}
\psi_{k}(1-\varepsilon)=\left(\frac{\sigma_{N-1}}{\sigma_{N}}\right)\left(\frac{\gamma}{2}\right) \varepsilon^{N+k-2}+\left(\frac{\sigma_{N-1}}{\sigma_{N}}\right)\left(\frac{\gamma(N-1)-B_{1}}{4}\right) \varepsilon^{N+k-1}+O\left(\varepsilon^{N+k}\right), \\
\psi_{k}(1+\varepsilon)=-\left(\frac{\sigma_{N-1}}{\sigma_{N}}\right)\left(\frac{\gamma}{2}\right) \varepsilon^{N+k-2}+\left(\frac{\sigma_{N-1}}{\sigma_{N}}\right)\left(\frac{\gamma(5-N-2 k)-B_{1}}{4}\right) \varepsilon^{N+k-1}+O\left(\varepsilon^{N+k}\right),
\end{array}
$$

and so (2)(i)-(ii) directly follow.

\section{References}

[1] L. Ambrosio, N. Gigli, G. Savaré, Gradient Flows in Metric Spaces and in the Space of Probability Measures, in: Lectures in Mathematics, Birkhäuser, 2005.

[2] G.E. Andrews, R. Askey, R. Roy, Special Functions, in: Encyclopedia of Mathematics and its Applications, vol. 71, Cambridge University Press, Cambridge, 1999.

[3] J. Bedrossian, N. Rodríguez, A.L. Bertozzi, Local and global well-posedness for aggregation equations and Patlak-Keller-Segel models with degenerate diffusion, Nonlinearity 24 (6) (2011) 1683-1714.

[4] S. Bian, J.-G. Liu, Dynamic and steady states for multi-dimensional Keller-Segel model with diffusion exponent $m>0$, Comm. Math. Phys. 323 (3) (2013) 1017-1070.

[5] A. Blanchet, V. Calvez, J.A. Carrillo, Convergence of the mass-transport steepest descent scheme for the subcritical Patlak-Keller-Segel model, SIAM J. Numer. Anal. 46 (2) (2008) 691-721.

[6] A. Blanchet, E.A. Carlen, J.A. Carrillo, Functional inequalities, thick tails and asymptotics for the critical mass Patlak-Keller-Segel model, J. Funct. Anal. 262 (5) (2012) 2142-2230.

[7] A. Blanchet, J.A. Carrillo, P. Laurençot, Critical mass for a Patlak-Keller-Segel model with degenerate diffusion in higher dimensions, Calc. Var. Partial Differential Equations 35 (2) (2009) 133-168.

[8] A. Blanchet, J.A. Carrillo, N. Masmoudi, Infinite time aggregation for the critical Patlak-Keller-Segel model in $\mathbb{R}^{2}$, Comm. Pure Appl. Math. 61 (10) (2008) 1449-1481.

[9] A. Blanchet, J. Dolbeault, B. Perthame, Two dimensional Keller-Segel model in $\mathbb{R}^{2}$ : optimal critical mass and qualitative properties of the solution, Electron. J. Differential Equations 2006 (44) (2006) 1-33. (electronic).

[10] M. Bonforte, J. Dolbeault, G. Grillo, J.L. Vázquez, Sharp rates of decay of solutions to the nonlinear fast diffusion equation via functional inequalities, Proc. Natl. Acad. Sci. USA 107 (38) (2010) 16459-16464.

[11] V. Calvez, J. Carrillo, Volume effects in the Keller-Segel model: energy estimates preventing blow-up, J. Math. Pures Appl. 86 (2006) 155-175.

[12] V. Calvez, J.A. Carrillo, Refined asymptotics for the subcritical Keller-Segel system and related functional inequalities, Proc. Amer. Math. Soc. 140 (10) (2012) 3515-3530.

[13] V. Calvez, J.A. Carrillo, F. Hoffmann, The geometry of diffusing and self-attracting particles in a one-dimensional faircompetition regime. Preprint arXiv:1612.08225.

[14] V. Calvez, L. Corrias, Blow-up dynamics of self-attracting diffusive particles driven by competing convexities, Discrete Contin. Dyn. Syst. Ser. B 18 (8) (2013) 2029-2050.

[15] V. Calvez, T.O. Gallouët, Particle approximation of the one dimensional Keller-Segel equation, stability and rigidity of the blow-up, Discrete Contin. Dyn. Syst. 36 (3) (2016) 1175-1208.

[16] E.A. Carlen, A. Figalli, Stability for a GNS inequality and the log-HLS inequality, with application to the critical mass Keller-Segel equation, Duke Math. J. 162 (3) (2013) 579-625.

[17] E. Carlen, M. Loss, Competing symmetries, the logarithmic HLS inequality and Onofri's inequality on $\mathbb{S}^{n}$, Geom. Funct. Anal. 2 (1992) 90-104. 
[18] J.A. Carrillo, D. Castorina, B. Volzone, Ground states for diffusion dominated free energies with logarithmic interaction, SIAM J. Math. Anal. 47 (1) (2015) 1-25.

[19] J.A. Carrillo, S. Hittmeir, B. Volzone, Y. Yao, Nonlinear aggregation-diffusion equations: Radial symmetry and long time asymptotics. Preprint arXiv:1603.07767.

[20] J.A. Carrillo, F. Hoffmann, E. Mainini, B. Volzone, Ground states in the diffusion-dominated regime. Preprint.

[21] J. Carrillo, R. McCann, C. Villani, Kinetic equilibration rates for granular media and related equations: entropy dissipation and mass transportation estimates, Rev. Mat. Iberoam. 19 (2003) 1-48.

[22] J. Carrillo, R. McCann, C. Villani, Contractions in the 2-Wasserstein length space and thermalization of granular media, Arch. Ration. Mech. Anal. 179 (2006) 217-263.

[23] J.A. Carrillo, G. Toscani, Asymptotic $L^{1}$-decay of solutions of the porous medium equation to self-similarity, Indiana Univ. Math. J. 49 (1) (2000) 113-142.

[24] P.-H. Chavanis, P. Laurençot, M. Lemou, Chapman-Enskog derivation of the generalized Smoluchowski equation, Phys. A 341 (1-4) (2004) 145-164.

[25] P.H. Chavanis, R. Mannella, Self-gravitating Brownian particles in two dimensions: the case of $N=2$ particles, Eur. Phys. J. B 78 (2) (2010) 139-165.

[26] L. Chen, J.-G. Liu, J. Wang, Multidimensional degenerate Keller-Segel system with critical diffusion exponent $2 n /(n+2)$, SIAM J. Math. Anal. 44 (2) (2012) 1077-1102.

[27] L. Chen, J. Wang, Exact criterion for global existence and blow up to a degenerate Keller-Segel system, Doc. Math. 19 (2014) 103-120.

[28] S. Childress, J. Percus, Nonlinear aspects of chemotaxis, Math. Biosci. 56 (1981) 217-237.

[29] T. Cieślak, P. Laurençot, Global existence vs. blowup in a one-dimensional Smoluchowski-Poisson system, in: Parabolic Problems, in: Progr. Nonlinear Differential Equations Appl., vol. 80, Birkhäuser/Springer Basel AG, Basel, 2011, pp. 95-109.

[30] L. Corrias, B. Perthame, H. Zaag, Global solutions of some chemotaxis and angiogenesis systems in high space dimensions, Milan J. Math. 72 (2004) 1-28.

[31] J. Dolbeault, B. Perthame, Optimal critical mass in the two dimensional Keller-Segel model in $\mathbb{R}^{2}$, C. R. Math. Acad. Sci. Paris 339 (2004) 611-616.

[32] H. Dong, The aggregation equation with power-law kernels: ill-posedness, mass concentration and similarity solutions, Comm. Math. Phys. 304 (3) (2011) 649-664.

[33] W. Jäger, S. Luckhaus, On explosions of solutions to a system of partial differential equations modelling chemotaxis, Trans. Amer. Math. Soc. 329 (1992) 819-824.

[34] R. Jordan, D. Kinderlehrer, F. Otto, The variational formulation of the Fokker-Planck equation, SIAM J. Math. Anal. 29 (1) (1998) 1-17.

[35] E. Keller, L. Segel, Initiation of slime mold aggregation viewed as an instability, J. Theoret. Biol. 26 (1970) $399-415$.

[36] E. Keller, L. Segel, Model for chemotaxis, J. Theoret. Biol. 30 (1971) 225-234.

[37] S. Kesavan, Topics in Functional Analysis and Applications, John Wiley \& Sons, Inc., New York, 1989.

[38] E. Lieb, M. Loss, Analysis, second ed., in: Graduate Studies in Math., vol. 14, Amer. Math. Soc., Providence, RI, 2001.

[39] J.-G. Liu, J. Wang, A note on $L^{\infty}$-bound and uniqueness to a degenerate Keller-Segel model, Acta Appl. Math. 142 (2016) $173-188$.

[40] T. Nagai, Blow-up of radially symmetric solutions to a chemotaxis system, Adv. Math. Sci. Appl. 5 (1995) 581-601.

[41] V. Nanjundiah, Chemotaxis, signal relaying and aggregation morphology, J. Theoret. Biol. 42 (1973) 63-105.

[42] F. Otto, The geometry of dissipative evolution equations: the porous medium equation, Comm. Partial Differential Equations 26 (1-2) (2001) 101-174.

[43] B. Perthame, Transport Equations in Biology, in: Frontiers in Mathematics, Birkhäuser, 2006.

[44] W. Rudin, Real and Complex Analysis, third ed., McGraw-Hill Book Co., New York, 1987.

[45] W. Sickel, Composition operators acting on Sobolev spaces of fractional order - a survey on sufficient and necessary conditions, in: Function Spaces, Differential Operators and Nonlinear Analysis (Paseky nad Jizerou, 1995), Prometheus, Prague, 1996, pp. 159-182.

[46] L. Silvestre, Regularity of the obstacle problem for a fractional power of the Laplace operator, Comm. Pure Appl. Math. 60 (1) (2007) 67-112.

[47] E.M. Stein, Singular Integrals and Differentiability Properties of Functions, in: Princeton Mathematical Series, No. 30, Princeton University Press, Princeton, N.J, 1970.

[48] G. Ströhmer, Stationary states and moving planes, in: Parabolic and Navier-Stokes Equations. Part 2, in: Banach Center Publ., vol. 81, Polish Acad. Sci. Inst. Math., Warsaw, 2008, pp. 501-513.

[49] Y. Sugiyama, Global existence in sub-critical cases and finite time blow-up in super-critical cases to degenerate Keller-Segel systems, Differential Integral Equations 19 (8) (2006) 841-876.

[50] Y. Sugiyama, Application of the best constant of the Sobolev inequality to degenerate Keller-Segel models, Adv. Differential Equations 12 (2) (2007) 121-144.

[51] Y. Sugiyama, The global existence and asymptotic behavior of solutions to degenerate quasi-linear parabolic systems of chemotaxis, Differential Integral Equations 20 (2) (2007) 133-180.

[52] Y. Sugiyama, H. Kunii, Global existence and decay properties for a degenerate Keller-Segel model with a power factor in drift term, J. Differential Equations 227 (1) (2006) 333-364.

[53] J.L. Vázquez, Smoothing and Decay Estimates for Nonlinear Diffusion Equations, in: Oxford Lecture Series in Mathematics and its Applications, vol. 33, Oxford University Press, Oxford, 2006, Equations of porous medium type.

[54] J.L. Vázquez, The Porous Medium Equation, in: Oxford Mathematical Monographs, The Clarendon Press, Oxford University Press, Oxford, 2007, Mathematical theory. 
[55] C. Villani, Topics in Optimal Transportation, in: Graduate Studies in Mathematics, vol. 58, American Mathematical Society, Providence, RI, 2003.

[56] Y. Yao, Asymptotic behavior for critical Patlak-Keller-Segel model and a repulsive-attractive aggregation equation, Ann. Inst. H. Poincaré Anal. Non Linéaire 31 (1) (2014) 81-101. 